\title{
STELLAR POPULATIONS IN THE CENTRAL 0.5 pc OF THE GALAXY. II. THE INITIAL MASS FUNCTION
}

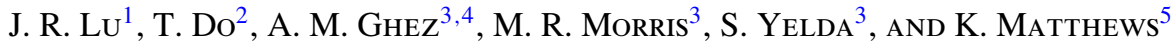 \\ ${ }^{1}$ Institute for Astronomy, University of Hawaii, Honolulu, HI 96822, USA; jlu@ ifa.hawaii.edu \\ ${ }^{2}$ Department of Physics and Astronomy, University of California, Irvine, CA 92697, USA; tuan.do@uci.edu \\ ${ }^{3}$ Department of Physics and Astronomy, University of California, Los Angeles, CA 90095-1547, USA; ghez@astro.ucla.edu, morris@astro.ucla.edu \\ ${ }^{4}$ Institute of Geophysics and Planetary Physics, University of California, Los Angeles, CA 90095-1565, USA \\ ${ }^{5}$ Division of Physics, Mathematics, and Astronomy, California Institute of Technology, MC 301-17, Pasadena, CA 91125, USA; kym@caltech.edu \\ Received 2012 September 21; accepted 2012 December 31; published 2013 February 1
}

\begin{abstract}
The supermassive black hole at the center of the Milky Way plays host to a massive, young cluster that may have formed in one of the most inhospitable environments in the Galaxy. We present new measurements of the global properties of this cluster, including the initial mass function (IMF), age, and cluster mass. These results are based on Keck laser-guide-star adaptive optics observations used to identify the young stars and measure their $K p$-band luminosity function as presented in Do et al. A Bayesian inference methodology is developed to simultaneously fit the global properties of the cluster utilizing the observations and extensive simulations of synthetic star clusters. We find that the slope of the mass function for this cluster is $\alpha=1.7 \pm 0.2$, which is steeper than previously reported, but still flatter than the traditional Salpeter slope of 2.35. The age of the cluster is between 2.5 and $5.8 \mathrm{Myr}$ with $95 \%$ confidence, which is a younger age than typically adopted but consistent within the uncertainties of past measurements. The exact age of the cluster is difficult to determine since our results show two distinct age solutions (3.9 Myr and 2.8 Myr) due to model degeneracies in the relative number of Wolf-Rayet and OB stars. The total cluster mass is between 14,000 and $37,000 M_{\odot}$ above $1 M_{\odot}$ and it is necessary to include multiple star systems in order to fit the observed luminosity function and the number of observed Wolf-Rayet stars. The new IMF slope measurement is now consistent with X-ray observations indicating a factor of 10 fewer X-ray emitting pre-main-sequence stars than expected when compared with a Salpeter IMF. The young cluster at the Galactic center is one of the few definitive examples of an IMF that deviates significantly from the near-universal IMFs found in the solar neighborhood.
\end{abstract}

Key words: Galaxy: center - infrared: stars - methods: statistical - stars: evolution - stars: luminosity function, mass function - stars: massive

Online-only material: color figures

\section{INTRODUCTION}

Young nuclear star clusters have now been found surrounding supermassive black holes (SMBHs) in a number of nearby galaxies (e.g., Lauer et al. 1998; Bender et al. 2005; Seth et al. 2006). The best studied young nuclear cluster (YNC) is at the center of our own Milky Way Galaxy, located only $8 \mathrm{kpc}$ away and surrounding an SMBH of mass $M \sim 4 \times 10^{6} M_{\odot}$ (Eckart \& Genzel 1997; Ghez et al. 1998, 2000, 2003, 2005, 2008; Genzel et al. 2000; Schödel et al. 2002, 2003; Eisenhauer et al. 2005; Gillessen et al. 2009a). The young stars are located within 1 pc of the SMBH and are thought to have formed as recently as 4-8 Myr ago (Paumard et al. 2006). The origin of the young stars, in such close proximity to the SMBH, is puzzling, given that the strong tidal forces in this region will shear apart typical molecular clouds before they can collapse to form stars. Thus, the $\mathrm{YNC}$ is a key laboratory for understanding whether and how stars form under such extreme conditions.

The YNC at the Galactic center has several observed properties that may help to determine its origin. To date, more than 100 young stars have been spectroscopically identified as OB supergiants, Wolf-Rayet (WR) stars, and, more recently, OB main-sequence stars (Allen et al. 1990; Krabbe et al. 1991, 1995; Blum et al. 1995; Tamblyn et al. 1996; Najarro et al. 1997; Ghez et al. 2003; Paumard et al. 2006; Bartko et al. 2010; Do et al. 2013). The young population appears to fall into three dynamical categories: (1) a well-defined clockwise rotating disk ranging from $0.03 \mathrm{pc}$ to at least $0.5 \mathrm{pc}$ with moderate eccentricities,
(2) an off-the-disk population in a more isotropic distribution over the same distances and also with moderate eccentricities, and (3) $\sim 10 \%$ cluster within $0.03 \mathrm{pc}$ of the black hole with high eccentricities of $\bar{e}=0.8$ (Genzel et al. 2000, 2003; Levin \& Beloborodov 2003; Paumard et al. 2006; Ghez et al. 2008; Lu et al. 2009; Gillessen et al. 2009b; Yelda 2012). These kinematic differences have led to some speculation that the young stars may have been formed in multiple episodes, although the outer two groups (1 and 2) have consistent stellar populations (Paumard et al. 2006). The inner group (3) was most likely dynamically injected, since the SMBH's tidal forces are too strong to permit star formation at these close distances. Initial theories suggested that this inner population was substantially older, as long times were needed to scatter inward a large number of binary systems that can interact with the SMBH and leave behind a star on a highly eccentric orbit with a small semimajor axis (e.g., Brown et al. 2007; Perets et al. 2007). However, more recent inclusion of additional dynamical effects suggests that the stars in the central region can be brought in more efficiently than initially thought (Löckmann et al. 2009; Madigan et al. 2011). Thus, it remains uncertain whether the inner group of young stars was originally part of the outer groups and born in the same star formation event. In the outer groups, there is some observational support for a possible warp in the clockwise disk (Bartko et al. 2009), a second face-on disk made of counterclockwise rotating stars (Genzel et al. 2003; Bartko et al. 2009), and for sub-clusters of stars both on and off the disk (Maillard et al. 2004; Schödel et al. 2005; Lu et al. 2005), although the 
statistical significance of these results is still debated (Lu et al. 2009; Yelda 2012). The total surface density profile of the young stars in the plane of the sky is $\Sigma \propto R^{-1}$ (Do et al. 2013) and substantially steeper in the disk plane $\Sigma_{\mathrm{CW} \text { disk }} \propto R^{-2}$. Early analysis of the bright stars $(K \leqslant 13)$ gave an age for the cluster of $6 \pm 2$ Myr based on the presence of WR stars and the proportions of WR stars to O stars (Paumard et al. 2006). In this same work, the observed number of WR and $\mathrm{O}$ stars and their luminosity function suggested that the total cluster mass is $10^{4} M_{\odot}$ and the initial mass function (IMF; $d N / d m \propto m^{\alpha}$ ) is top-heavy with a slope significantly flatter than Salpeter $(1955, \alpha=2.35)$. However, these results were limited by the lack of sensitivity to less massive, main-sequence stars $\left(K>13, M<20 M_{\odot}\right)$. Deeper spectroscopic studies initially showed that the luminosity function for the young stars appears to have a sharp turnover at $K=13.5$, suggesting that the present-day mass function is extremely top-heavy with a slope of $\alpha=0.45 \pm 0.3$ (Bartko et al. 2010). A top-heavy IMF is also supported by the Chandra observations of the region, given that lower mass young stars should still be coronally active and emitting copious X-rays that are not detected (Nayakshin \& Sunyaev 2005).

Several possible models for the origin of the young stars have been proposed and the two presently supported by observations include (1) formation in situ in a massive self-gravitating molecular disk (Levin \& Beloborodov 2003) or (2) disruption of an infalling massive cluster formed much further away (Gerhard 2001). In situ formation models invoke the build-up of a gas disk surrounding the SMBH that reached a critical mass $\left(\sim 10^{4} M_{\odot}\right)$ approximately $6 \mathrm{Myr}$ ago such that local self-gravity within the disk became sufficient to overcome the tidal shear and the disk collapsed along the vertical direction and formed stars (Levin \& Beloborodov 2003; Kolykhalov \& Syunyaev 1980; Shlosman \& Begelman 1989; Morris \& Serabyn 1996; Sanders 1998; Goodman 2003; Nayakshin \& Cuadra 2005). If such a disk was built up slowly, the gas and resulting young stars would largely be on circular orbits, which does not appear to be the case observationally (Lu et al. 2009; Bartko et al. 2009). Modified in situ formation models include the rapid infall of a single massive molecular cloud or the collision of two infalling clouds to produce non-circular orbits and the on-disk and off-disk populations (Alexander et al. 2007; Cuadra et al. 2008; Sanders 1998; Vollmer \& Duschl 2001; Nayakshin et al. 2007; Hobbs \& Nayakshin 2009). The surface density of stars formed in situ in a slowly-built gas disk may be steep $\left(\Sigma \propto R^{-2}\right)$; however, the surface density resulting from a cloud-cloud collision is less well constrained. For infalling cluster scenarios, the young stars are formed a few parsecs away in a massive young star cluster that spirals in via dynamical friction and disrupts in the central parsec (Gerhard 2001). In order for such a cluster to migrate into the central parsec within only a few million years, the cluster must be very massive and centrally concentrated and perhaps even host an intermediate-mass black hole (IMBH) at its center (Kim \& Morris 2003; Portegies Zwart et al. 2003; McMillan \& Portegies Zwart 2003; Gürkan \& Rasio 2005; Hansen \& Milosavljević 2003; Kim et al. 2004). Such a scenario would produce the on-disk population and a flatter surface density profile $\left(\Sigma \propto R^{-0.75}\right)$, with the off-disk population resulting from subsequent dynamical perturbations (Haas et al. 2011; Baruteau et al. 2011). In situ formation models are favored based on the density profiles and available time scales; however, no single model completely explains all of the observed properties. One important prediction of current in situ formation models is that the IMF should be very top-heavy due to the extreme temperatures, pressures, densities, and ambient radiation fields present in the central parsec (Nayakshin et al. 2006; Nayakshin 2006; Alexander et al. 2007, 2008; Cuadra et al. 2008). However, a number of model parameters are still very uncertain (e.g., gas infall rate, temperature, pressure, and cooling time) and a wide variety of IMFs are still possible.

The IMF, age, and mass of the young nuclear star cluster at the Galactic center is of particular interest both for understanding the origin of the young stars and exploring star formation under extreme conditions. Past observations of a turnover in the nearinfrared luminosity function and the low total X-ray emission apparently support a top-heavy IMF. However, new observations presented in our companion paper, Do et al. (2013, Paper I), show a luminosity function that does not turn over, but continues to rise, warranting a new analysis of the mass function.

In this work, we compare the near-infrared photometry of the spectroscopically identified young stars in Paper I to models of star clusters in order to determine the age, mass function, and total mass of the young nuclear star cluster. Details on the observational sample and measurements are presented in Section 2. Synthetic clusters are generated using stellar evolution and atmosphere models to produce individual stars as they would appear at the Galactic center in Section 3.1. In Section 3.2 we present a Bayesian inference method for fitting the observed data with synthetic clusters to determine the cluster's properties and uncertainties. Results in Section 4 show that the present-day mass function is slightly flatter than a Salpeter IMF and rule out a mass function as top-heavy as previously claimed. We also find a younger age and our modeling requires the inclusion of binaries and multiple star systems to adequately fit the luminosity function.

\section{OBSERVED DATA AND SAMPLE}

Observations and data used in this paper are described in Paper I. Our data sample includes all stars with a non-zero probability of being a young, early-type $\operatorname{star}\left(p_{\text {yng }}>0\right)$. For each star, the measurements consist of a $K p$ magnitude corrected for differential extinction $\left(K_{\Delta A}^{\prime}\right)$, a $K p$ uncertainty $\left(\sigma_{K^{\prime}}\right)$, the probability of youth $\left(p_{\text {yng }}\right)$, and an indicator for whether the star is a WR star. Only stars with $K_{\Delta A}^{\prime} \leqslant 15.5$ are included. The modeling presented in this work utilizes these individual measurements rather than a binned $K p$ luminosity function (KLF). However for illustrative purposes, Figure 1 shows our binned KLF compared with a model KLF for a cluster with an age of $6 \mathrm{Myr}$, extinction of $A_{K s}=2.7$, distance of $8 \mathrm{kpc}$, and an IMF slope of $\alpha=0.45$, consistent with previously published best-fit cluster parameters (Bartko et al. 2010). The discrepancy between our observed KLF and this model motivates the work presented below as we attempt to derive new best-fit cluster parameters and uncertainties.

In our sample, we have chosen to include young stars at all radii, including the central 0 .'8 region immediately around the SMBH. There are some suggestions that the stars in this central region were not formed in the most recent starburst, but rather in an earlier event. The brightest early-type star in this region, S0-2, is a B0-B2.5 V star with an age of less than 15 Myr based on its measured temperature, gravity, and luminosity (Martins et al. 2008). The other early-type stars in this region have spectral features consistent with main-sequence stars (Eisenhauer et al. 2005) and the oldest age for a main-sequence star at $K p=15.5$ $\left(A_{K s}=2.7, d=8 \mathrm{kpc}\right.$ ) is less than $20 \mathrm{Myr}$. Given these age constraints and the large uncertainties in the theoretical 


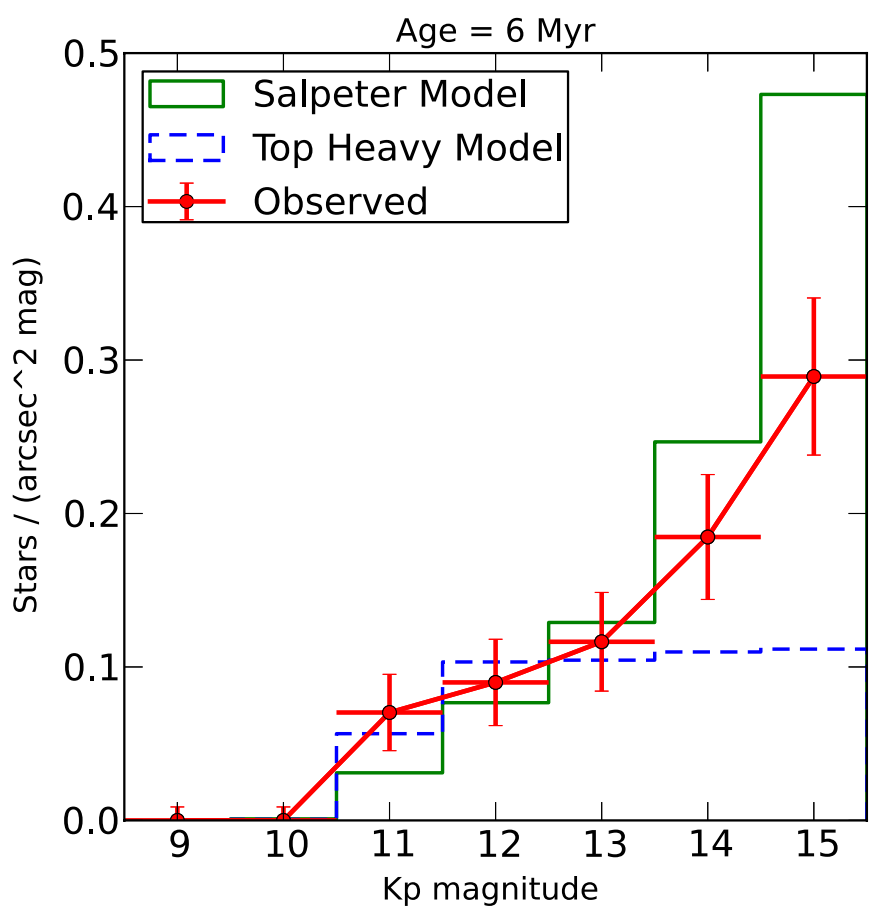

Figure 1. Observed $K p$ luminosity function (KLF) for early-type stars in the Galactic center nuclear cluster (Paper I) compared with previously proposed model KLFs. The observed $K p$ magnitudes (red) are corrected for differential extinction to a common extinction value of $A_{K s}=2.7$. A model KLF is shown for published best-fitting cluster parameters (blue) with an IMF slope of $\alpha=0.45$, age of $6 \mathrm{Myr}$, and a distance of $8 \mathrm{kpc}$ (Bartko et al. 2010). For comparison, a model KLF is also shown for a similar cluster with a Salpeter IMF slope of $\alpha=2.35$ (green).

scattering efficiencies, it remains plausible that the young stars in the central region of our sample are the same age as the stars at larger radii. Furthermore, as described in Paper I, including or discarding the stars within a radius of $0{ }^{\prime \prime} 8$ does not significantly change the shape of the KLF.

\section{MODELING THE OBSERVED CLUSTER}

While the early-type stellar population in the central parsec of the Galaxy can be divided into several different kinematic subgroups (Paumard et al. 2006; Ghez et al. 2008; Lu et al. 2009; Bartko et al. 2009; Gillessen et al. 2009b), it is not yet clear whether these subgroups have different origins, or whether all the young stars formed from the same starburst and were differentiated via subsequent dynamical processes. Therefore, we choose to analyze the entire population of young stars as a single starburst cluster and to estimate the cluster's mass function, age, and total mass.

The traditional approach to deriving a mass function would be to construct a binned KLF from the observed $K p$ magnitudes, assume a distance and extinction, and use a mass-luminosity relation from models of stellar evolution and atmospheres to convert from observed magnitudes to initial stellar masses. This approach has several shortcomings, including arbitrarily choosing bin sizes, neglecting uncertainties on stellar brightness, not accounting for multiple systems, and correlations in the cluster age, mass, and IMF (Maíz Apellániz \& Úbeda 2005; Maíz Apellániz 2008; Maíz-Apellániz 2009). More recent and rigorous statistical methods, particularly those using Bayesian inference, provide flexibility and produce robust estimates of the most probable cluster parameters and their uncertainties (e.g., Allen et al. 2005). We have developed a Bayesian inference methodology for deriving the physical properties of an observed young star cluster using simulations of synthetic clusters. The process of simulating a synthetic young cluster is described in Section 3.1 and the full Bayesian methodology, implementation, and testing is described in Sections 3.2, 3.3, and 3.4, respectively. Readers wishing to skip the detailed description of the modeling may proceed to Section 4 where the resulting best-fit cluster properties and uncertainties are presented.

\subsection{Synthetic Clusters}

Model young star clusters are produced assuming an instantaneous starburst at time $t$, at distance $d$, with an observed cluster mass $M_{\mathrm{cl} \text {,obs. }}$. The IMF of the cluster is described as a single power law given by the probability density function

$$
\begin{aligned}
\frac{d N}{d m} & \propto p(m)=\frac{(1-\alpha) m^{-\alpha}}{m_{\max }^{1-\alpha}-m_{\min }^{1-\alpha}} \quad[\alpha \neq 1] \\
& =\frac{m^{-\alpha}}{\ln m_{\max }-\ln m_{\min }} \quad[\alpha=1]
\end{aligned}
$$

where $\alpha$ is allowed to vary and has the value $\alpha=2.35$ in the case of a typical IMF for the high-mass end of a solar-neighborhood population (Salpeter 1955). In addition to these free parameters, a number of fixed parameters are also needed to fully model the cluster. First, the extinction is fixed to $A_{K s}=2.7$ as we will compare our model data to observations that have been corrected for differential extinction to this value. The metallicity is fixed to $Z=0.02$, which is roughly solar. Observations suggest the young Galactic center population is consistent with solar iron abundance; but may be as high as $2 \times$ solar and seems enhanced in $\alpha$-elements (Carr et al. 2000; Ramírez et al. 2000; Najarro et al. 2004; Cunha et al. 2007; Martins et al. 2008). The choice to keep metallicity fixed to solar is also motivated by the lack of complete stellar evolution and atmosphere models at other metallicities and abundance ratios. Section 5 discusses possible uncertainties associated with restricting our analysis to solar metallicity. The range of masses we consider is set as $m_{\min }=$ $1 M_{\odot}$ and $m_{\max }=150 M_{\odot}$ and is kept fixed throughout the analysis. This is justified as our observations do not include stars below $10 M_{\odot}$ due to sensitivity limits and stars above $150 M_{\odot}$ have already exploded for clusters older than 3 Myr. Thus the currently observed stars have no power to constrain the low or high mass cutoffs and a more expansive mass range would not impact our results. For each star drawn from the IMF, we must also consider whether it has companions (Sagar \& Richtler 1991; Kroupa 1995; Goodwin \& Kroupa 2005; Thies \& Kroupa 2007; Maíz-Apellániz 2009; Weidner et al. 2009). High mass stars in the Galactic disk are known to have a high multiplicity fraction, perhaps even $100 \%$ (Kobulnicky \& Fryer 2007) and the multiplicity fraction and the companion star fraction, which is the mean number of companions per primary, are dependent on the mass of the primary star in the multiple system (e.g., Lafrenière et al. 2008). The Galactic center cluster is modeled allowing multiple systems with a mass-dependent multiplicity fraction (MF) and companion star fraction (CSF). The functional form of how the MF and CSF vary with primary mass is still very uncertain even for solar-neighborhood young clusters. We empirically derive this relation by compiling MF and CSF measurements in young clusters from the literature and assuming that the MF and CSF follow a power-law distribution (see the Appendix for the derivation). Each star drawn from the 
Table 1

Model Cluster Parameters

\begin{tabular}{llcc}
\hline \hline Parameter & Vary? & Description & Prior \\
\hline$\alpha$ & Free & IMF slope between $m_{\min }$ and $m_{\max }$ & Uniform from $[0.10,3.35]$ \\
$\log t$ & Free & $\log _{10}($ age of the young cluster in years $)$ & Gaussian with $\log t=6.78, \sigma=0.18$ from $[6.20,6.70]$ \\
$M_{\text {cl,obs }}$ & Free & Initial mass of the young cluster & Uniform from $\left[10^{3}, 10^{5}\right] M_{\odot}$ \\
$d$ & Free & Distance to the young cluster & Gaussian with $\bar{d}=8.096 \mathrm{kpc}, \sigma=0.483 \mathrm{kpc}$ from $[6.793,9.510]$ \\
$m_{\min }$ & Fixed & IMF minimum stellar mass & $1 M_{\odot}$ \\
$m_{\max }$ & Fixed & IMF maximum stellar mass & $150 M_{\odot}$ \\
$Z$ & Fixed & Metallicity of the young cluster & 0.20 (roughly solar) \\
$A_{K s}$ & Fixed & Extinction to the young cluster & 2.7 \\
MF_amp & Fixed & MF function's amplitude & 0.44 \\
MF_index & Fixed & MF function's power-law index & 0.51 \\
CSF_amp & Fixed & CSF function's amplitude & 0.50 \\
CSF_index & Fixed & CSF function's power-law index & 0.45 \\
CSF_max & Fixed & Maximum value for CSF & 3 \\
$\beta$ & Fixed & Power-law index for $q$ distribution & -0.4
\end{tabular}

IMF with mass $m$ is determined to be in a multiple system and assigned a number of companions based on

$$
\begin{gathered}
\operatorname{MF}(m)=0.44\left(\frac{m}{1 M_{\odot}}\right)^{0.52} \quad \text { always } \leqslant 1 \\
\operatorname{CSF}(m)=0.48\left(\frac{m}{1 M_{\odot}}\right)^{0.49} \quad \text { always } \leqslant 3 .
\end{gathered}
$$

The mass of the companion stars is drawn from a power-law probability distribution on the mass ratio, $q$ :

$$
p(q)=\left(\frac{1+\beta}{1-q_{\mathrm{lo}}^{1+\beta}}\right) q^{\beta}
$$

where $\beta=-0.4$ and the lowest allowed mass ratio is $q_{\mathrm{lo}}=0.01$. Our choice of $\beta$ is consistent with observations of local massive stars in young OB associations, although values ranging from $\beta=-0.6$ to 0.4 would also be within $68 \%$ confidence intervals allowed by observations ${ }^{6}$ (Kobulnicky \& Fryer 2007; Kiminki \& Kobulnicky 2012). Luminosity functions are hardly impacted by this choice of $q_{\text {lo }}$ since lower mass ratios do not significantly increase the total system luminosity (Kouwenhoven et al. 2009). Allowing for multiple systems will have the largest effect at the bright end of the luminosity function where magnitude bins that were unpopulated by individual stars are filled in with multiple star systems composed of massive, bright primaries and companions (Maíz-Apellániz 2009; Weidner et al. 2009). In Section 3.4, simulated clusters are used to show that incorporating multiple systems is essential and that failing to include them produces poor fits and incorrect ages and IMF slopes. For a cluster with a desired cluster mass of $M_{\mathrm{cl}, \mathrm{obs}}$, stars are simulated until the sum of all stellar masses, including companions, exceeds $M_{\mathrm{cl}, \mathrm{obs}}$ and then the last simulated star is thrown out if that would bring the synthesized cluster mass closer to the desired $M_{\mathrm{cl} \text {,obs }}$ (Haas 2010). A complete list of free and fixed parameters we use to produce synthetic star clusters is presented in Table 1.

Conversion from stellar masses to synthetic photometry requires the use of stellar evolution and atmosphere models. At the suspected age of the young cluster (4-8 Myr), stars with masses

\footnotetext{
6 The resulting companion star masses are substantially higher than randomly drawing companions from the IMF.
}

in excess of 30-60 $M_{\odot}$ are evolving off the main-sequence and stellar evolution models with rotation are found to best describe populations of blue supergiants and WR stars (Meynet $\&$ Maeder 2003). We use the grid of Geneva models with an initial rotation speed of $300 \mathrm{~km} \mathrm{~s}^{-1}$ provided by Meynet \& Maeder (2003), which only extends down to $9 M_{\odot}{ }^{7}$ In these models, WR stars are identified using the criteria suggested in Meynet \& Maeder (2003): $T_{\text {eff }}>10^{4} \mathrm{~K}$ and the mass fraction of hydrogen at the surface is $X_{s}<0.4$. To extend to lower masses along the main sequence and also capture pre-mainsequence evolution at younger ages, the Geneva grid is merged with the grid of models provided by Siess et al. (2000) that extends up to $7 M_{\odot}$. For both grids, a suite of isochrones is generated at logarithmic ages of $\log t=6.0-7.4$ with steps of $\Delta \log t=0.01$ and each isochrone samples mass more finely than $\Delta m=0.005 M_{\odot}$. The two isochrones are then merged and interpolated over the gap between the two models $\left(7-9 M_{\odot}\right)$. An example of the merging process for $\log t=6.8$ is shown in Figure 2. The resulting isochrones provide a mapping from the simulated stellar masses to their luminosities $(L)$, effective temperatures $\left(T_{\text {eff }}\right)$, and surface gravities $(g)$.

The physical parameters for each star are converted into observable brightness at $K p$-band using model stellar atmospheres. Again, there is no single set of atmosphere models that spans the full range of effective temperatures and surface gravities. For hot stars with $T_{\text {eff }}>7000 \mathrm{~K}$, atmosphere models by Castelli \& Kurucz (2003) are used for all non-WR stars. For temperatures of $4000 \mathrm{~K}<T_{\text {eff }}<7000 \mathrm{~K}$, we use the NextGen models of Hauschildt et al. (1999) and for $T_{\text {eff }}<4000 \mathrm{~K}$ we use the updated NextGen models with improved AMES opacities (Allard et al. 2000). The grids do not intersect exactly at the transition temperatures; however, synthetic broadband photometry differs by $\lesssim 2 \%$ in the $K$-band, which is less than our photometric precision, so no interpolation between the different atmosphere models is done. All downloaded atmosphere models were reformatted to work with the pysynphot Python package in order to perform interpolations within each grid and generate synthetic spectra at specific temperatures, metallicities, and gravities. The synthetic spectra are flux calibrated to what would be observed at Earth without extinction by multiplying by $(R / d)^{2}$, where $R$ is the radius of the star and $d$ is the distance. Then the synthetic spectra are reddened using the Galactic Center extinction law

\footnotetext{
7 Ekström et al. (2012) contains an updated grid of Geneva models with rotation extending down to lower masses and with rotation speeds other than $300 \mathrm{~km} \mathrm{~s}^{-1}$; however, they were not available at the time of our analysis.
} 


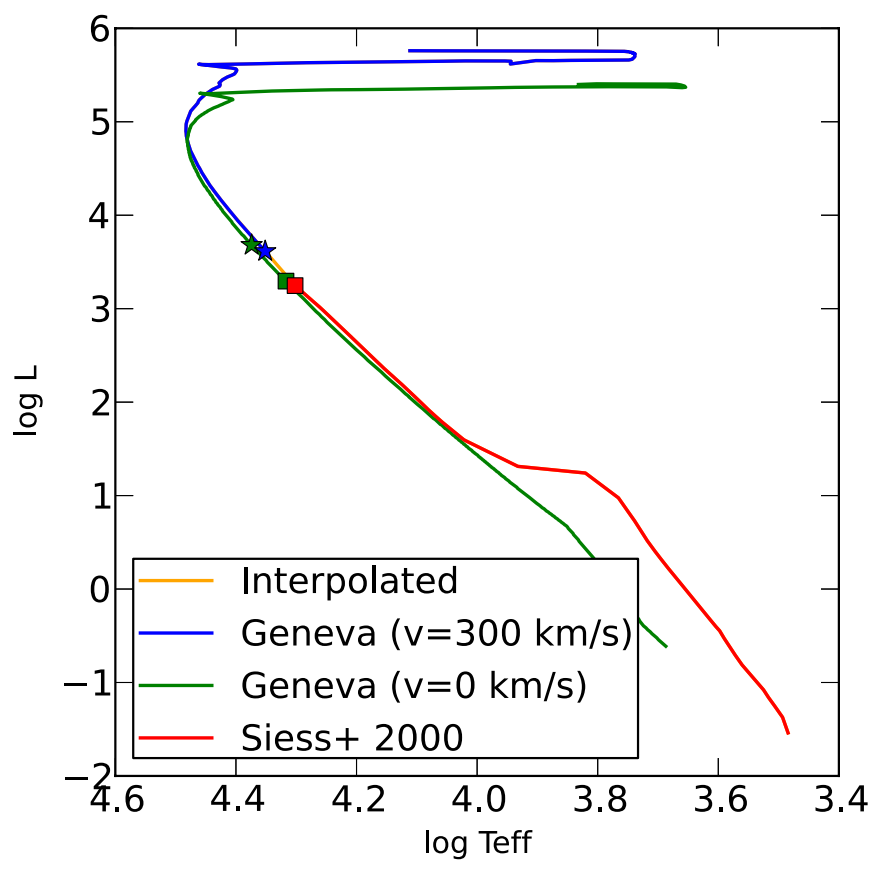

Figure 2. 6 Myr luminosity-effective temperature isochrones from various stellar evolution models used to construct and test a combined grid of stellar evolution models that spans ages from 1 to $25 \mathrm{Myr}$ and the full range of stellar masses. Geneva models without rotation spanning $0.8-120 M_{\odot}$ are shown in green as a baseline (Schaller et al. 1992). The observed post-main-sequence evolution of massive stars at this age is best described with models that include rotation. The isochrone from Geneva models with rotation, shown in blue, differs significantly in luminosity. Models with rotation only extend down to $9 M_{\odot}$ (blue star and green star). Models provided by Siess et al. (2000) are used for main sequence and pre-main-sequence models below $7 M_{\odot}$ and are shown in red ( $7 M_{\odot}$ point also shown as a red square and green square). The blue and red isochrones are merged and interpolated over the gap to construct final isochrones, shown in orange, used in our cluster modeling.

from Nishiyama et al. (2009) and convolved with transmission profiles for a typical Mauna Kea atmosphere and for the $K p$ filter in NIRC2. The final synthetic spectra are integrated using pysynphot to produce broadband $K p$ photometry as would be seen from the telescope. An example mass $-K p$ magnitude relation is shown in Figure 5 (left panel) and would predict that S0-2, with a $K p=14.39$ after correcting to $A_{K s}=2.7$, would have a mass of $\sim 18 M_{\odot}$, which is consistent with previous estimates based on the effective temperature and surface gravity derived from detail spectral analysis (Martins et al. 2008).

WR stars require special consideration as their atmospheres are windy and clumpy. Models of stellar evolution for WR stars can predict when stars enter and exit the WR phase, surface abundances, and the luminosities and temperatures at the base of the atmosphere. However, this is insufficient to uniquely define what will be observed due to differences in wind velocities, mass loss rates, and clumping factors (e.g., Hamann et al. 2008). Put another way, there is no unique mass-magnitude relationship for WR stars. Model atmospheres do exist for WR stars, but they require observations of the wind velocities and mass-loss rates and still do not uniquely predict the interior properties of the star, such as temperature and gravity at the base of the atmosphere (Martins et al. 2007). Therefore, the observed magnitudes of the WR stars cannot be used in a straightforward manner and we exclude them from the analysis of the luminosity function. The number of WR stars, $N_{\mathrm{WR}}$, relative to the number of OB stars is still a powerful constraint on the age and mass of the cluster and we incorporate $N_{\mathrm{WR}}$ into our Bayesian analysis.

\subsection{Bayesian Methodology}

A Bayesian inference approach is used to determine the physical properties of the YNC at the Galactic center. This methodology is generally applicable to the analysis of other star clusters. The advantages of Bayesian inference for astronomical applications are highlighted in Press (1997) and have been widely adopted in cosmology (Hobson et al. 2010). Bayesian techniques are beginning to be used more widely in the study of all types of star clusters (Selman et al. 1999; Allen et al. 2005; Converse \& Stahler 2008; De Gennaro et al. 2009; van Dyk et al. 2009; Espinoza et al. 2009; Rizzuto et al. 2011). The analysis presented here differs from previous work in several ways. First, single-band photometric information is combined with some spectroscopic information that identify which stars are WR stars. In our case, only single band photometry is necessary since extinction maps are available from past multi-band photometry (Schödel et al. 2010). Second, the IMF is assumed to have the functional form of a truncated power law, although other functional forms can easily be inserted. This differs from some Bayesian treatments that attempt to derive the masses of the individual stars allowing for infinite variation in the shape of the mass function (Converse \& Stahler 2008; van Dyk et al. 2009; De Gennaro et al. 2009). Third, we allow for mass-dependent multiplicity fractions and high-order multiple systems, which are important at the high mass end of the mass function. Fourth, we include all candidate young stars and weight them by their probability of youth in order to use all available information and accurately account for incompleteness. ${ }^{8}$ Finally, instead of using Markov Chain Monte Carlo (MCMC) techniques to perform the Bayesian inference, we find that better solutions result from nested sampling techniques (Feroz \& Hobson 2008; Feroz et al. 2009). This Bayesian approach provides detailed probability distributions for the mass function slope and other cluster parameters given limited observations. It also avoids traditional biases from binning and neglecting photometric errors and accounts for stochastic sampling of stellar masses. Our full methodology is described below in more detail.

We start at the beginning, with Bayes equation

$$
p(\Theta \mid D)=\frac{p(D \mid \Theta) p(\Theta)}{p(D)}
$$

where $D$ is the observed data, $\Theta$ is the model, $p(D \mid \Theta)$ is the likelihood of observing the data given some model, $p(\Theta)$ captures the prior knowledge on the model parameters, and $p(D)$ is a normalizing factor known as the evidence. The likelihood function and the prior distributions then give posterior probability distributions for the model parameters, $p(\Theta \mid D)$. The model, $\Theta$, is defined by the set of free and fixed parameters given in Table 1.

The data, $D$, is constructed from the sample of candidate young stars $\left(p_{\text {yng }}>0\right)$ brighter than $K p=15.5$ as described in Section 2. The WR stars are separated out and only the observed number of WR stars, $N_{\mathrm{WR}}$, is included in our analysis, since the initial masses of WR stars cannot be determined based on their luminosity alone. For all the other non-WR stars, measurements of the $K p$ brightness and error are used along

\footnotetext{
8 A significant number of the candidate young stars were spectral-typed manually and were assigned $100 \%$ probability of youth. See Do et al. (2013) for a complete discussion.
} 
with the probability that each star is young, $p_{\text {yng. Although }}$ we observe $N_{\text {obs }}$ non-WR stars, not all of them are young. We estimate the number of young OB stars, $N_{\mathrm{OB}}$, as the sum of the observed non-WR stars, weighted by their probability of youth: $N_{\mathrm{OB}}=\sum_{i=1}^{N_{\text {obs }}} p_{\mathrm{yng}, i}$. The resulting measurements that make up the data are then

$$
D=\left[N_{\mathrm{WR}}, \mathbf{k}_{\mathrm{obs}}, N_{\mathrm{OB}}\right]
$$

where $\mathbf{k}_{\mathrm{obs}}$ is the set of $\left\{k_{i}, \sigma_{k_{i}}, p_{\mathrm{yng}, i}\right\}$ measurements for the $N_{\text {obs }}$ non-WR stars observed.

The likelihood function is composed of three independent probabilities: (1) the likelihood of observing the set of $K p$ magnitudes, $\left\{k_{i}\right\}$, and uncertainties, $\left\{\sigma_{k_{i}}\right\}$, which effectively captures the shape of the KLF; (2) the likelihood of observing $N_{\mathrm{OB}} \mathrm{OB}$ stars, which captures the normalization of the KLF; and (3) the likelihood of observing $N_{\mathrm{WR}}$ WR stars:

$$
p(D \mid \Theta)=p\left(\mathbf{k}_{\mathrm{obs}} \mid \Theta\right) \cdot p\left(N_{\mathrm{OB}} \mid \Theta\right) . \cdot p\left(N_{\mathrm{WR}} \mid \Theta\right) .
$$

To derive the first term, we use an unbinned approach such that the likelihood is the multiplication of the individual stars' probabilities, weighted by the probability of youth,

$$
p\left(\mathbf{k}_{\mathrm{obs}} \mid \Theta\right)=\prod_{i=1}^{N_{\mathrm{obs}}} p\left(k_{i} \mid \Theta\right)^{p_{\mathrm{yng}, i}}
$$

The probability for a star to have some $K p, p\left(k_{i} \mid \Theta\right)$, is given by the probability distribution derived from synthetically "observing" a simulated young cluster. First, the cluster is simulated with model parameters describing the age $(t)$, IMF slope $(\alpha)$, cluster mass $\left(M_{\mathrm{cl}, \mathrm{obs}}\right)$, and the cluster distance $(d)$,

$$
p(k \mid \Theta)_{\text {intrinsic }}=\text { Simulated Yng Cluster }\left(t, \alpha, M_{\mathrm{cl}, \mathrm{obs}}, d\right) .
$$

These free parameters along with their priors are given in Table 1 . The resulting $K p$ photometry from the simulated stars in the model cluster are binned finely ( $0.1 \mathrm{mag}$ bins) to produce a probability distribution for the intrinsic $K p$ luminosities. For small numbers of stars, stochastic sampling effects can lead to an inaccurate estimate of the probability distribution. Therefore, in order to maximize accuracy and reduce the total number of clusters that need to be simulated, all model young clusters are simulated with a total mass of $10^{6} M_{\odot}$. The shape of the $K p$ luminosity function does not change with cluster mass and the number of WR and non-WR stars simply scales linearly with total cluster mass. The intrinsic probability distribution for $K p$ luminosities is multiplied by the imaging completeness curve, $C(k)$, truncated at $K p=15.5$ to match the data, and normalized to give the final probability distribution,

$$
p(k \mid \Theta)_{\text {observed }}=\frac{p(k \mid \Theta)_{\text {intrinsic }} \cdot C(k)}{\int_{0}^{15.5} p(k \mid \Theta)_{\text {intrinsic }} \cdot C(k) d k} .
$$

Finally, the measured photometric error is incorporated by modeling the observed magnitude as $k_{i}=k^{\prime}+\epsilon$ where $\epsilon$ is drawn from a normal distribution with zero mean and a standard deviation of $\sigma_{k_{i}}$. The resulting probability of observing $k_{i}$ for a given star is then given by

$$
p\left(k_{i} \mid \Theta\right)=\int^{\infty} p\left(k^{\prime} \mid \Theta\right)_{\text {observed }} \frac{1}{\sqrt{2 \pi \sigma_{k_{i}}^{2}}} e^{\frac{-\left(k^{\prime}-k_{i}\right)^{2}}{2 \sigma_{k_{i}}^{2}}} d k^{\prime},
$$

which is the convolution of the model KLF and the error probability distribution. The resulting probabilities for each star feed into Equation (9) to calculate the first term of the total likelihood in Equation (8). In the second and third terms of the likelihood, the number of OB and WR stars scales linearly with the mass of the young cluster, which was verified with simulations. Thus the same simulated young clusters used to produce $p(k \mid \Theta)_{\text {intrinsic }}$ also give the expected number of WR and $\mathrm{OB}$ stars after scaling linearly with cluster mass,

$$
\begin{gathered}
\aleph_{\mathrm{WR}}\left(M_{\mathrm{cl}, \mathrm{obs}}\right)=\aleph_{\mathrm{WR}}\left(10^{6} M_{\odot}\right)\left(\frac{M_{\mathrm{cl}, \mathrm{obs}}}{10^{6} M_{\odot}}\right) \\
\aleph_{\mathrm{OB}}\left(M_{\mathrm{cl}, \mathrm{obs}}\right)=\aleph_{\mathrm{OB}}\left(10^{6} M_{\odot}\right)\left(\frac{M_{\mathrm{cl}, \mathrm{obs}}}{10^{6} M_{\odot}}\right)
\end{gathered}
$$

where $\aleph_{\mathrm{WR}}$ and $\aleph_{\mathrm{OB}}$ are the expectation values (means) for the number of WR and non-WR stars, respectively. The likelihood of observing $N_{\mathrm{WR}}$ and $N_{\mathrm{OB}}$ are then taken as a Poisson distributions

$$
\begin{aligned}
& p\left(N_{\mathrm{WR}} \mid \Theta\right)=\frac{\aleph_{\mathrm{WR}}(\Theta)^{N_{\mathrm{WR}}} e^{\aleph_{\mathrm{WR}}(\Theta)}}{N_{\mathrm{WR}} !} \\
& p\left(N_{\mathrm{OB}} \mid \Theta\right)=\frac{\aleph_{\mathrm{OB}}(\Theta)^{N_{\mathrm{OB}}} e^{\aleph_{\mathrm{OB}}(\Theta)}}{N_{\mathrm{OB}} !} .
\end{aligned}
$$

\subsection{Sampling Posterior Probability Distributions with Multinest}

The resulting posterior probability distribution for the model parameters cannot be calculated analytically. Traditionally, Monte Carlo techniques are used to produce a representative sample of points from the posterior probability distribution. The most commonly used method is the MCMC. However, MCMC methods may have difficulty converging or fully mapping probability space when the probability distributions that are being sampled are multi-modal or highly degenerate and curved. An alternative method is nested sampling (Skilling 2004), such as in the publicly available multi-modal nested sampling code, MultiNest (Feroz \& Hobson 2008; Feroz et al. 2009). This algorithm has been successfully demonstrated in cosmology, galaxy evolution, and gravitational wave problems (e.g., Bridges et al. 2009; Kilbinger et al. 2010; Martinez et al. 2011; Veitch \& Vecchio 2010) and is less computationally expensive and more accurate, in some cases, than MCMC methods. We performed tests on simulated clusters using traditional MCMC techniques using both Metropolis-Hastings and Hitand-Run step methods (PyMC package) and compared the overall computation time and accuracy with the MultiNest code. MultiNest took 5-10 times less computation time and produced more accurate results in all of our simulated clusters (see Section 3.4 for more details). Therefore, we utilized MultiNest in our analysis of the young nuclear star cluster's IMF.

MultiNest uses a fixed number of points per iteration to sample parameter space and calculate the Bayesian evidence at each point. In successive iterations, the same number of points are concentrated into smaller and smaller volumes centered around the most probable regions. This process continues until the evidence no longer changes by more than a specified tolerance value. We use 1000 points and an evidence tolerance of 0.3 in order to sample parameter space well and run efficiently. We also enabled multi-modal searches as several simulated clusters could clearly be fit by several distinct sets of model parameters. 

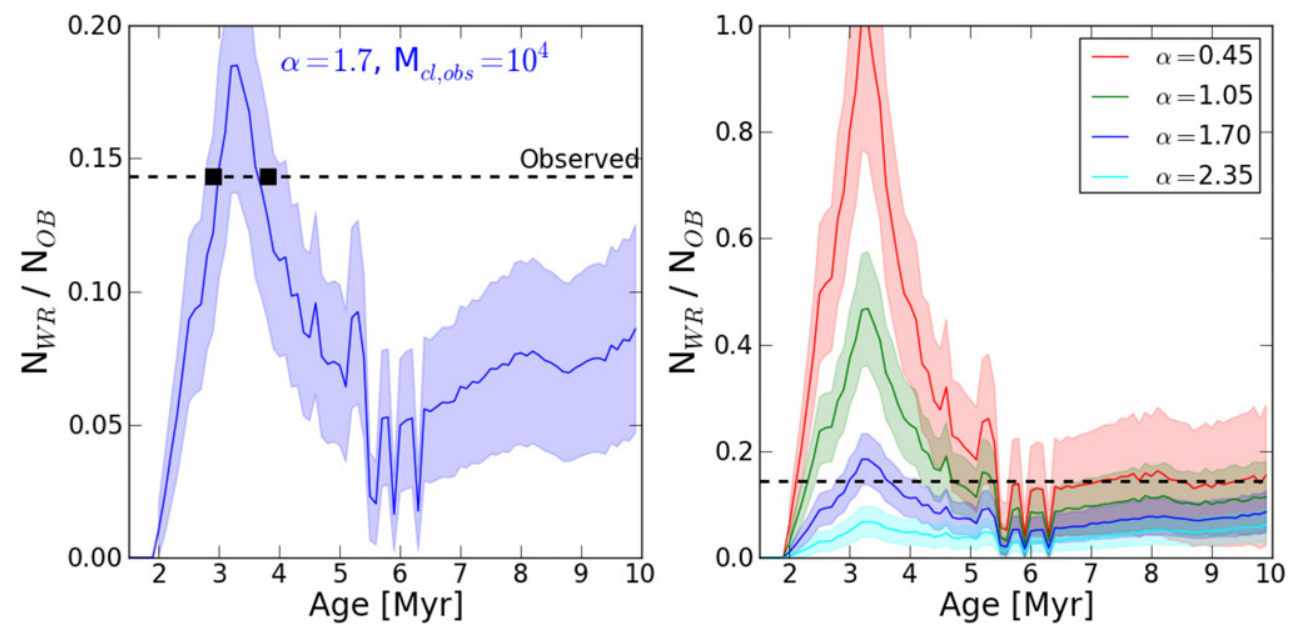

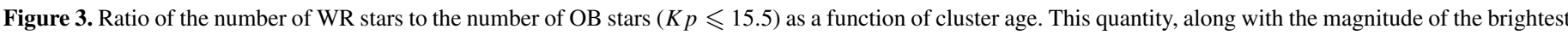

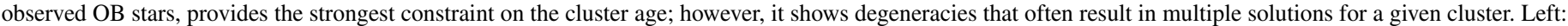

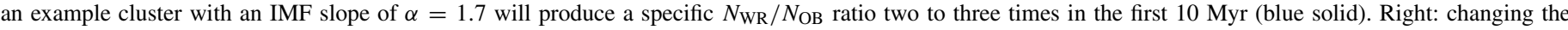

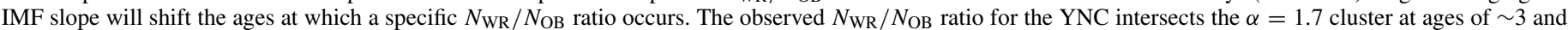

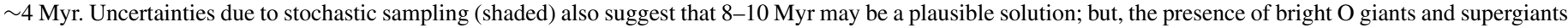
makes these larger ages unlikely.

(A color version of this figure is available in the online journal.)

\subsection{Testing on Simulated Clusters}

To test the speed and accuracy of our Bayesian methodology and codes, clusters were simulated and synthetically "observed" that had different ages, IMFs, and multiplicity properties. The synthetic "observations" were then fit with the Bayesian inference techniques described above and the resulting posterior probability distribution functions were examined to see whether they produced results consistent with the input cluster parameters. All clusters were simulated at a distance of $8 \mathrm{kpc}$, an extinction of $A_{K s}=2.7$, and with a cluster mass aimed at producing a similar number of OB stars to our observed data set. Photometric errors for simulated cluster stars are randomly drawn from the distribution of empirical errors from the Galactic center observations. Tests were performed to (1) understand degeneracies in fitting a cluster's age, (2) determine the impact of multiplicity, and (3) explore whether we recover both top-heavy and normal IMF slopes.

$$
\text { 3.4.1. Age }
$$

A cluster's age is often one of the most difficult parameters to constrain, especially at young ages and when only high-mass main-sequence and post-main-sequence stars are observed. The number of WR stars and the ratios of WR to OB stars can be a precise indicator of age; however, there are also cases where degeneracies produce several possible solutions (Paumard et al. 2006). To illustrate these degeneracies we simulate clusters at a range of ages from 1.5 to $10 \mathrm{Myr}$ and examine the ratio of $N_{\mathrm{WR}}$ to $N_{\mathrm{OB}}$. Note that $N_{\mathrm{OB}}$ includes all OB stars down to $K p=15.5$, which is equivalent to B1-B2 V. Figure 3 shows that $N_{\mathrm{WR}} / N_{\mathrm{OB}}$ initially rises around $2 \mathrm{Myr}$, peaks at $3 \mathrm{Myr}$, and falls to a minimum around $6 \mathrm{Myr}$, which is set almost entirely by the rise and fall of $N_{\mathrm{WR}}$. After $6 \mathrm{Myr}$, the ratio rises slightly again as $N_{\mathrm{WR}}$ remains relatively constant, but $N_{\mathrm{OB}}$ begins to drop. If we observe an $N_{\mathrm{WR}} / N_{\mathrm{OB}}$ ratio of 0.125 , then there are two possible ages: 2.9 Myr and 3.8 Myr, assuming an IMF slope of $\alpha=1.7$. This degeneracy is not entirely independent of the IMF slope (Figure 3, right panel). In order to explore how our Bayesian methodology handles this age degeneracy, we produce 10 different realizations of a cluster, all with the same input parameters $\left(t=4 \mathrm{Myr}, \alpha=1.7, M_{\mathrm{cl}, \mathrm{obs}}=10^{4} M_{\odot}, d=8 \mathrm{kpc}\right.$, single stars). Each cluster is then fit using the Bayesian inference methods described in Section 3.2. The resulting probability distributions for the age are shown in Figure 4 and multiple age solutions are almost always found. We note that in these one-dimensional (1D) marginalized probability distributions, the input cluster age is not always the highest peak. However, the input age is always recovered within the $99 \%$ confidence interval and with much higher confidence if each peak is treated as an independent solution in a multi-modal distribution. Given the results of our testing, all further age results will be reported using multi-modal solutions, if necessary.

\subsubsection{Multiplicity}

The need to incorporate multiplicity when determining a cluster's IMF is often discussed (Kroupa 1995; Goodwin \& Kroupa 2005; Weidner et al. 2009) but rarely implemented due to incomplete knowledge of multiplicity fractions, number of companions, companion mass functions, and how these all scale with primary mass (see the Appendix and references therein). The presence of multiple systems influences the bright end of the KLF as illustrated in Figure 5 by populating the brightest magnitude bins $(K p<11)$ with sources that would otherwise be much fainter single stars. We tested two possible scenarios to explore the impact of multiplicity in our analysis. First, a cluster was simulated with only single stars and analysis was done with and without allowing multiple systems in the fit. Second, a cluster was simulated with multiple systems and, again, analysis was done with and without allowing multiple systems in the fit. Both clusters had an IMF slope of $\alpha=2.35$, an age of $4 \mathrm{Myr}$, and a mass of $10^{4} M_{\odot}$, although similar tests were performed with different IMF slope and age combinations and the results are robust. Figure 6 shows the results for the simulated cluster containing only single stars. Incorrectly fitting this cluster with multiple systems produces extremely biased estimates for the cluster age and only slight biases in the IMF slope and cluster mass. Without prior information, it is difficult to discern that an inaccurate multiplicity model is being used. Figure 7 shows the results for the simulated cluster 


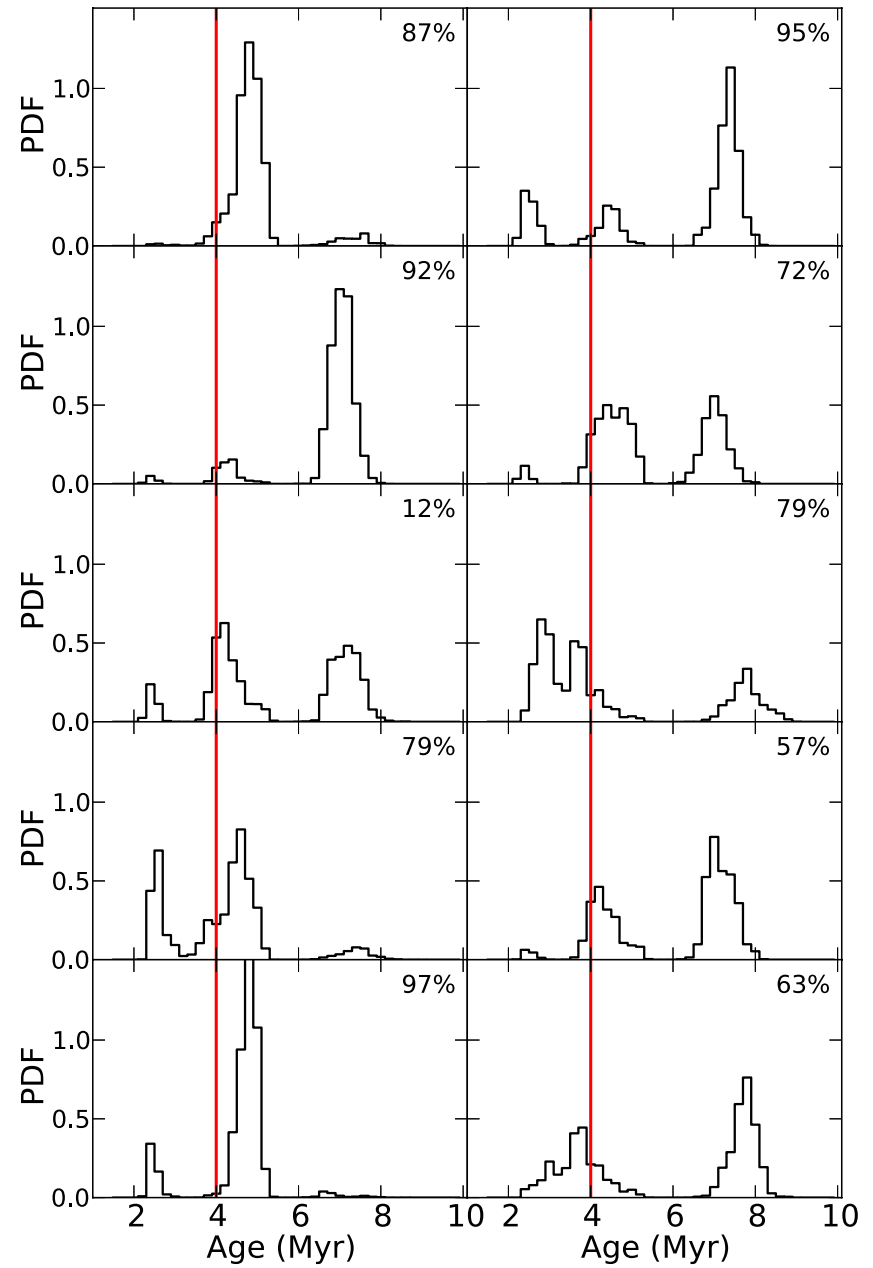

Figure 4. Test-clusters' age posterior probability distributions for 10 different realizations of a cluster with $\alpha=1.7, t=4 \mathrm{Myr}, d=8 \mathrm{kpc}, M_{\mathrm{cl}, \mathrm{obs}}=10^{4} M_{\odot}$, and only single stars. The input age of $4 \mathrm{Myr}$ is marked (vertical red) and the confidence interval that only includes $4 \mathrm{Myr}$ is reported in the top right corner of each panel. The confidence interval is calculated by sorting the probability distribution and integrating starting with the highest bins and proceeding until the bin including $4 \mathrm{Myr}$ is reached. The input age always falls within the $99 \%$ confidence interval. If each peak is treated as an independent solution, the input age is recovered with even greater confidence.

(A color version of this figure is available in the online journal.)

containing multiple systems. Incorrectly fitting this cluster with single stars produces extremely biased estimates for nearly all the parameters. Even without prior information, it is easy to discern that the single-star model is inaccurate since the distance tends toward unphysical low values. Multiple systems in clusters produce $K p$ magnitudes that are brighter than is allowed in the model clusters with only single stars at $8 \mathrm{kpc}$. Thus the only way to produce such bright systems is to move the cluster closer in distance. Shifting the cluster to younger ages is also a possibility; but such young clusters do not give the correct number of WR stars. The largest error is made when using single-star model clusters to fit to clusters with multiple systems. Thus, we choose to fit the observed data with simulated clusters containing multiple systems. All additional tests described below used simulated clusters containing multiple systems and were fit with multiples.

\subsubsection{IMF Slope}

Our Bayesian inference methodology shows no systematic biases with respect to the IMF slope as shown in the following
Table 2

Cluster Properties and Uncertainties

\begin{tabular}{lccc}
\hline \hline $\begin{array}{l}\text { Cluster } \\
\text { Properties }\end{array}$ & $\begin{array}{c}\text { Expectation } \\
\text { Value }\end{array}$ & $\begin{array}{c}68 \% \\
\text { Interval }\end{array}$ & $\begin{array}{c}95 \% \\
\text { Interval }\end{array}$ \\
\hline Solution 1: Age $>3.3 \mathrm{Myr}$ & & & \\
$\quad$ Age $(\mathrm{Myr})$ & 4.2 & $3.6-4.8$ & $3.4-6.1$ \\
$\quad$ IMF slope $(\alpha)$ & 1.7 & $1.5-1.9$ & $1.1-2.1$ \\
$\quad$ Integrated Mass $\left(10^{3} M_{\odot}\right)$ & 10.1 & $8.1-11.6$ & $7.2-18.5$ \\
$\quad$ Distance $(\mathrm{kpc})$ & 7.9 & $7.6-8.3$ & $7.3-8.6$ \\
Solution 2: Age $\leqslant 3.3 \mathrm{Myr}$ & & & \\
$\quad$ Age (Myr) & 2.8 & $2.6-3.1$ & $2.4-3.3$ \\
IMF slope $(\alpha)$ & 1.8 & $1.6-1.9$ & $1.5-2.1$ \\
Integrated Mass $\left(10^{3} M_{\odot}\right)$ & 8.8 & $7.7-9.5$ & $6.8-10.5$ \\
$\quad$ Distance $(\mathrm{kpc})$ & 7.9 & $7.6-8.2$ & $7.3-8.5$ \\
Total & & & \\
$\quad$ Age $(\mathrm{Myr})$ & 3.6 & $2.8-4.3$ & $2.5-5.8$ \\
IMF slope $(\alpha)$ & 1.7 & $1.5-1.9$ & $1.2-2.1$ \\
Integrated Mass $\left(10^{3} M_{\odot}\right)$ & 9.5 & $7.9-10.6$ & $7.1-16.7$ \\
Distance $(\mathrm{kpc})$ & 7.9 & $7.6-8.3$ & $7.3-8.6$ \\
\hline
\end{tabular}

Notes. The integrated masses correspond to that within the field of view of the survey (see Paper I for details) between 1 and $150 M_{\odot}$. The estimated mass for the entire $\mathrm{YNC}$ is $2-4$ times larger.

tests on synthetic clusters. Two clusters were simulated with an age of $6 \mathrm{Myr}$ and with IMF slopes of $\alpha=2.35$ (Salpeter) and $\alpha=0.45$ (top-heavy reported by Bartko et al. 2010). The Salpeter-like cluster has an input mass of $10,000 M_{\odot}$ and the top-heavy cluster has an input mass of $40,000 M_{\odot}$ in order to produce $\sim 100$ non-WR stars with $K p \leqslant 15.5$; similar to our observed data set. Figure 8 shows the output posterior probability distributions for the IMF slope, age, and cluster mass for the two simulated clusters. The input IMF slope falls well within the $68 \%$ confidence interval of the posterior probability density function. A handful of similar cluster tests were performed with different ages and masses and the input and output IMF slopes always agree within the $68 \%$ confidence region and there appears to be no significant bias to either higher or lower IMF slopes.

\section{RESULTS}

The observed $K p$ magnitudes, their uncertainties, and the number of WR and OB stars were used in Bayesian inference to determine the cluster's physical properties. Figure 9 shows the resulting 1D posterior probability distributions for the four free parameters in the model (cluster age, mass, distance, and IMF slope). The model assumes that multiple systems are present with the properties described in Section 3.1 and the Appendix. Several parameters show correlations, including a moderate correlation between the cluster age and IMF slope (Figure 10). The correlation between the cluster mass and the age or IMF slope is a consequence of the age-IMF slope relationship since, at older ages, the most massive stars have disappeared and the cluster mass must increase to match the observed numbers of stars brighter than $K p=15.5$. The posterior probability distributions are the most accurate representation of the results; however, we also present "best-fit" values in Table 2 represented by the expectation value and $68 \%$ and $95 \%$ Bayesian confidence intervals ${ }^{9}$ of the marginalized 1D posterior probability density functions. The resulting age distribution is multi-modal. Therefore, we report solutions from three possible samples: (1) ages greater than $3.3 \mathrm{Myr}$, (2) ages less than or

\footnotetext{
9 Central credible intervals.
} 

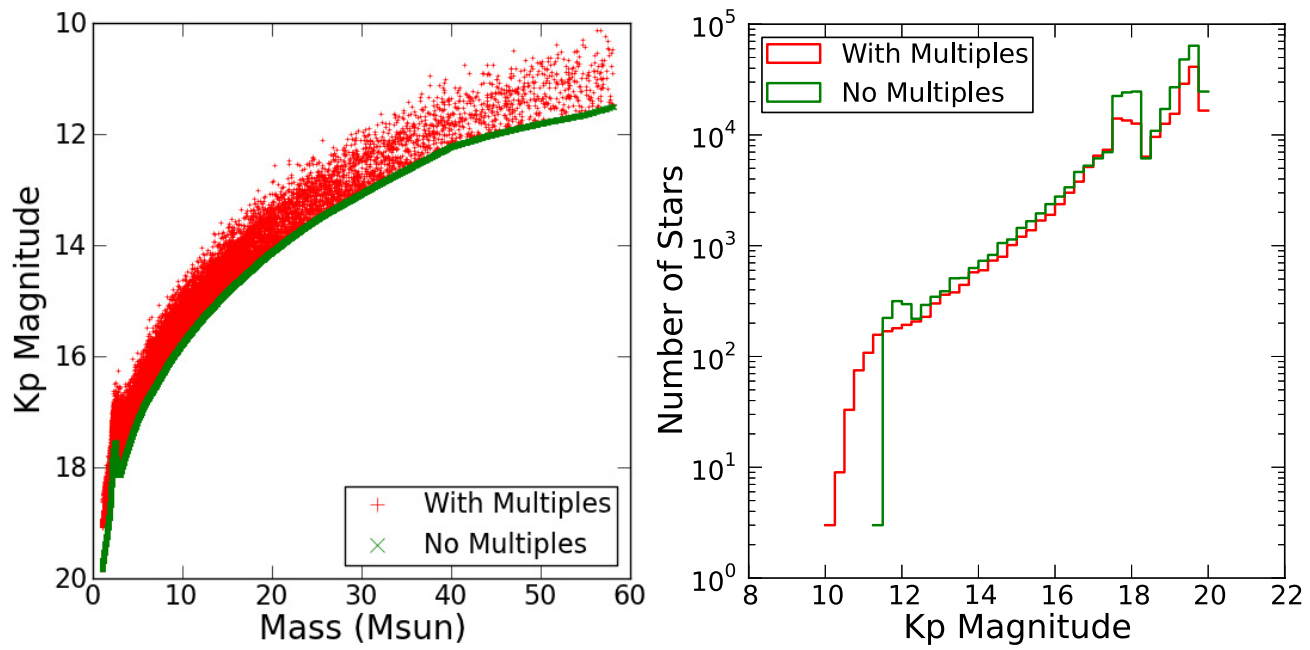

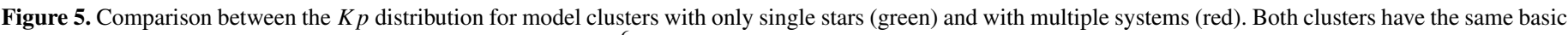

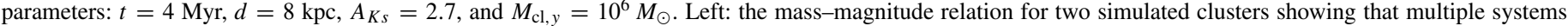

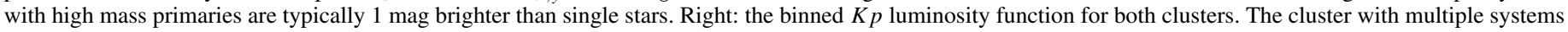
extends to higher $K p$ magnitudes than does the cluster with only single stars. We also note that the bump at $K p \sim 18$ is the pre-main-sequence turn-on.

(A color version of this figure is available in the online journal.)

equal to 3.3 Myr, and (3) the complete posteriors, The age boundaries for the two modal solutions were estimated based on the minima between the two peaks. We report all parameters for these three solutions; however, all solutions have similar $\alpha, M_{\mathrm{cl}, \text { obs }}$, and $d$ distributions. We will adopt Solution 1 in the remainder of the paper as it contains the most probable solution (with the maximum likelihood) and contains the bulk of the probability density. Solution 1 is also favored over the smaller age in Solution 2 based on more detailed spectroscopic analyses that show the WR star subtypes and the supergiant nature of the brightest OB stars favor ages $\gtrsim 4$ Myr (Paumard et al. 2006; Martins et al. 2007). A comparison of the observed and model KLFs and mass functions from the inferred parameters of Solution 1 is shown in Figure 11. The complete posteriors for the number of predicted WR and OB stars are also shown in Figure 12 and are compared with the observed number of WR and OB stars. The overall fit is good given the theoretical uncertainty in the evolution of massive stars and the unknown mapping between progenitor mass and WR phases.

For the IMF slope, recall that a flat prior from 0.5 to 3.5 was adopted; but the posterior distribution is strongly peaked at $1.7 \pm$ 0.2 . The slope is flatter than a typical Salpeter slope and slopes of 2.35 or greater are ruled out at $3.8 \sigma(99.98 \%)$. The slope is inconsistent with the previously reported top-heavy slope of 0.45 (Bartko et al. 2010) at the $3.8 \sigma$ level (99.98\%). Our sample of young stars includes those within $r<0$ '. 8 ; however, the results are very similar even when these stars are excluded (Figure 13), as is done in Bartko et al. 2010.

The cluster mass is $10,100 M_{\odot}(68 \%$ interval: 8100 $11,600 M_{\odot}$ ) between 1 and $150 M_{\odot}$ and is well constrained by the data, given that the posterior distribution is significantly different from the prior. The cluster age is also significantly different from the prior and shows multiple peaks. The most probable solution falls at 3.9 Myr (68\% interval: 3.6-4.8 Myr); although the second peak at $2.8 \mathrm{Myr}$ is still highly probable. This multi-modal behavior is a result of the rapid changes in the number of WR stars and the ratio of WR to OB stars in very short periods of time (Figure 3). Although the distance is also a free parameter, the resulting probability distribution mainly reflects the prior shown in the dashed line. This is reassuring since, in our testing, incorrect model assumptions (e.g., fitting single star models to data with multiple systems) often led to skewed distance distributions, which we do not observe using the multiple systems model.

The cluster mass and the numbers of WR and OB stars are based only on the young stellar population within the field of view observed for this study, which has incomplete azimuthal coverage. This does not represent the entire cluster mass of the young nuclear star cluster. The total cluster mass can be estimated by extrapolating beyond the field of view in this study, in several different ways. First, we use the assumption that the cluster is spherically symmetric and has a surface density profile that falls as $R^{-\Gamma}$ with $\Gamma=0.9$ (Paper I). This yields a surface density weighted coverage of $27 \%$ and a coveragecorrected total cluster mass of $\sim 37,000 M_{\odot}$ within a projected radius of 13 .' 65 and above $1 M_{\odot}$. The above assumption fails to account for known kinematic structures in the young population, including a highly inclined disk containing as much as $50 \%$ of the young stars. Furthermore, the above mass estimate assumes a constant IMF at all radii, which is unlikely given the mass segregation that will occur over 4 Myr. As an alternative, we instead make an empirically based estimate of the completeness by taking the ratio of the number of young stars with $K p<$ 13 within our field of view to the same number reported in Paumard et al. (2006), which we assume is $100 \%$ complete out to $14^{\prime \prime}$. This yields a coverage completeness of $71 \%$ and a total cluster mass of $\sim 14,000 M_{\odot}$ above $1 M_{\odot}$. Thus, we estimate the total cluster mass to be in the range of 14,000-37,000 $M_{\odot}$ above $1 M_{\odot}$. We note that the total cluster mass depends on the low-mass cutoff as $M_{\mathrm{cl}} \cdot\left(m_{\mathrm{lo}} / 1 M_{\odot}\right)^{-1.7}$; and, while we have assumed $1 M_{\odot}$, the lowest-mass young stars we observe are $\sim 8 M_{\odot}$. In order to improve the constraints on the total cluster mass, more sensitive and azimuthally complete spectroscopic surveys at large radii are necessary.

The above results are derived from modeling clusters with multiple systems; however, for completeness, we also show the resulting posterior probability distributions for a single-star model in Figure 14. It is clear that the fit is poor. The bias to very small distances indicates that the brightest $\mathrm{OB}$ stars combined with the number of WR stars cannot be accurately fit 

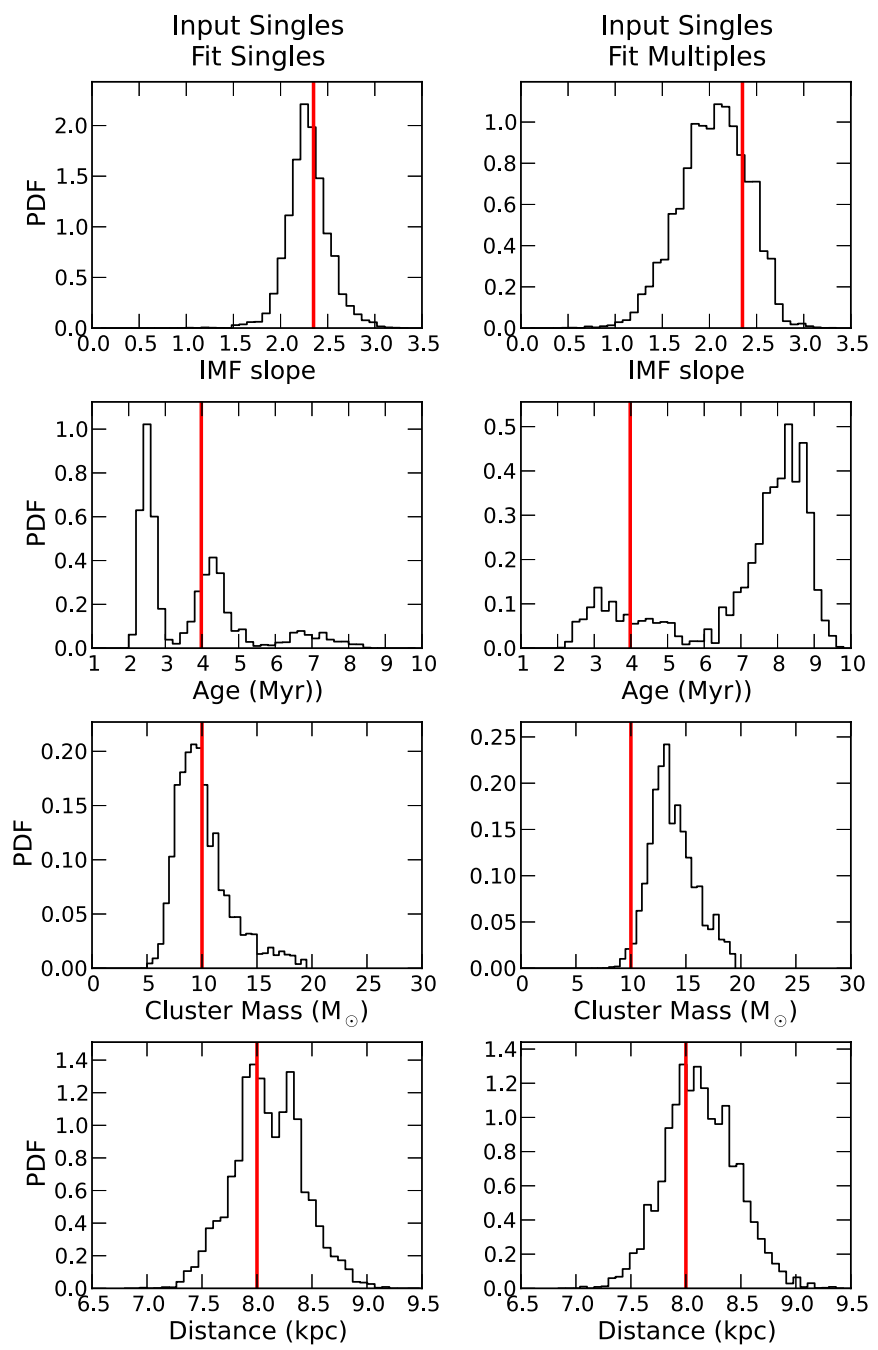

Figure 6. Test-cluster's marginalized 1D posterior probability density functions (PDFs) for a simulated cluster containing only single stars that is subsequently fit allowing only single stars (left) or multiple systems (right). The input values for the cluster's IMF slope, age, mass, and distance are shown as a vertical red line in each panel. For a cluster containing only single stars, fitting with multiple systems produces extremely biased estimates for the cluster age and only slight biases in the IMF slope and cluster mass. Without prior information, it is difficult to determine whether an inaccurate multiplicity model is being used.

(A color version of this figure is available in the online journal.)

with a single star model. Our modeling indicates that multiple star systems are necessary to fit the observed stellar population. However, we caution that the multiplicity properties were fixed to solar neighborhood values that may not be applicable to the YNC. Both the star formation process and subsequent dynamical processing may impact multiplicity.

\section{DISCUSSION}

The young nuclear star cluster at the Galactic center, at least along the plane of the well-defined clockwise disk, has an IMF slope in the range $\alpha=1.5-1.9$ (68\% confidence interval) for stellar masses above $\sim 10 M_{\odot}$. This is significantly flatter than clusters in the Milky Way disk and field $(\alpha=2.35)$. Our measured IMF is in good agreement with X-ray observations showing that X-ray emission from pre-main-sequence stars is reduced by a factor of 10 compared with what is expected from observations of the Orion Nebular Cluster, scaled to the age
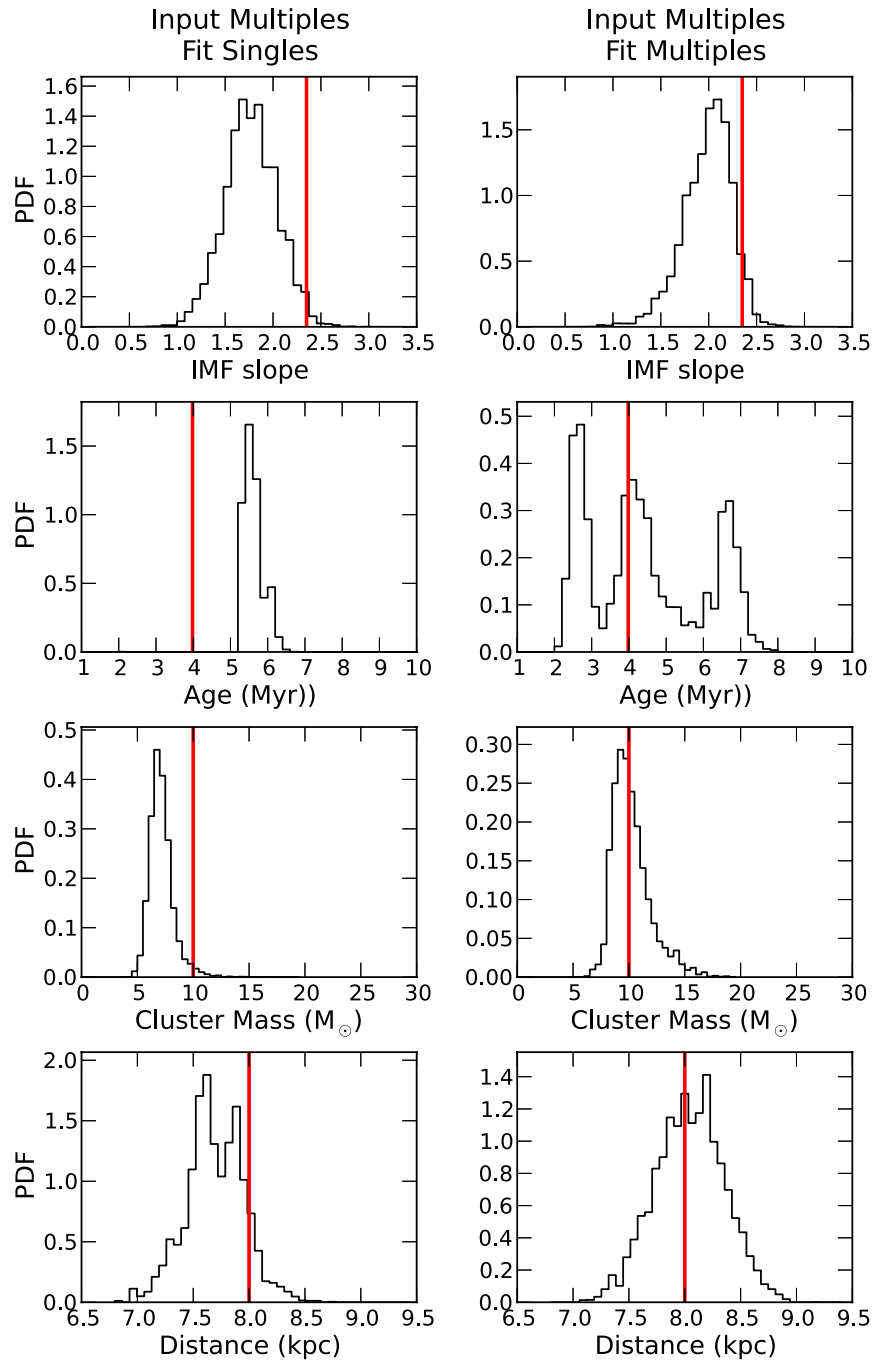

Figure 7. Test-cluster's marginalized 1D posterior PDFs for a simulated cluster containing multiple star systems that is subsequently fit allowing only single stars (left) or multiple systems (right). The input values for the cluster's IMF slope, age, mass, and distance are shown as a red line in each panel. For a cluster containing multiple systems, fitting with single stars produces extremely biased estimates for nearly all the parameters. Without prior information, it is easy to discern that an inaccurate multiplicity model is being used since the distance tends toward unphysically low values. Multiple systems in clusters produce bright systems that are brighter than is allowed in the model clusters with only single stars at $8 \mathrm{kpc}$. Thus the only way to produce such bright systems is to move the cluster closer in distance.

(A color version of this figure is available in the online journal.)

and distance of the Galactic center (Nayakshin \& Sunyaev 2005). Extrapolating our measured IMF slope down to lower masses produces a factor of 4-15 fewer X-ray emitting stars $\left(0.5-3.0 M_{\odot}\right)$ than would be expected for a slope of $\alpha=2.35$.

An extremely top-heavy IMF is inconsistent with our observations at high significance, in contradiction to previous observations (Bartko et al. 2010). An extensive discussion of the possible observational and analysis differences between our work and previous work is presented in Paper I. Here we reiterate a few key differences. The Bartko et al. (2010) field of view extends predominantly north, out of the plane of the well-defined clockwise disk of young stars. They also remove all young stars in the central $0^{\prime} .8$ and outside $12^{\prime \prime}$. In the work presented here, the spectroscopic window in which young stars are identified extends from the center to the east-southeast, along the well defined clockwise disk structure. The entire population of young 

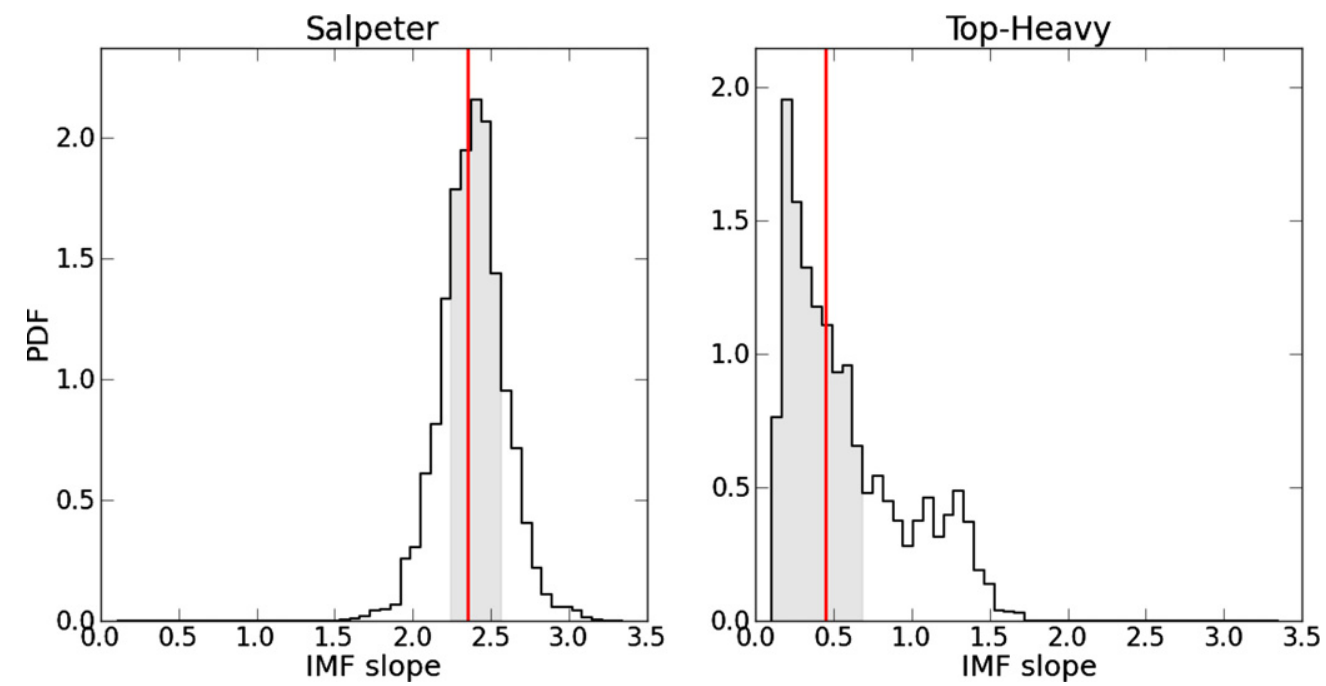

Figure 8. Test-cluster's marginalized 1D posterior PDFs for a simulated cluster with IMF slope $=2.35$ (left) and IMF slope $=0.45$ (right). The input values for the cluster's IMF slope, age, mass, and distance are shown as a vertical red lines in each panel. The gray shaded regions represent the $68 \%$ confidence interval for the output probability distributions and all of the input values fall within this interval. The confidence intervals are calculated by first finding the peak in the probability distribution and then stepping away from the peak until the integrated probability reaches $68 \%$.

(A color version of this figure is available in the online journal.)
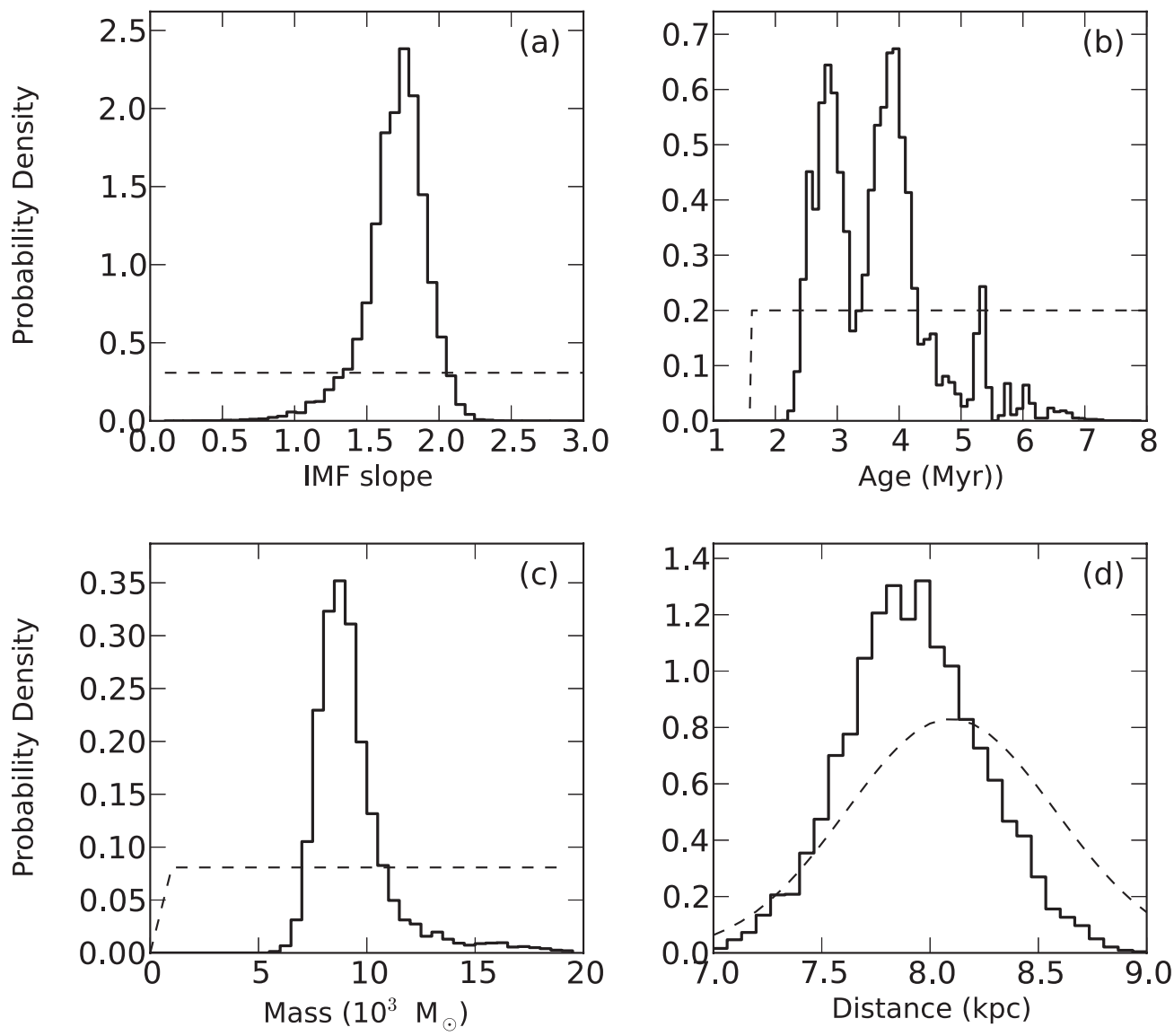

Figure 9. Observed YNC's marginalized 1D posterior PDFs for the cluster properties. The histograms show results from the Multinest Bayesian analysis including multiple systems. The dashed black lines show the prior probability distributions used. The resulting constraints on the IMF slope (panel (a)), the cluster age (panel (b)) and the cluster mass (panel (c)) are significant compared with the prior PDFs used. The distance constraint (panel (d)) is weak and is largely a reflection of the prior.

stars, including those within 0.8 of the SMBH, is included in the analysis. We assume that all the young stars were formed in a single star formation event with the same physical conditions giving rise to a constant IMF for all the kinematic subgroups.
As discussed in Sections 1 and 2, the stars in this innermost region were dynamically injected and it still appears to be theoretically possible that they originated from the most recent starburst that produced the YNC. Furthermore, restricting our 

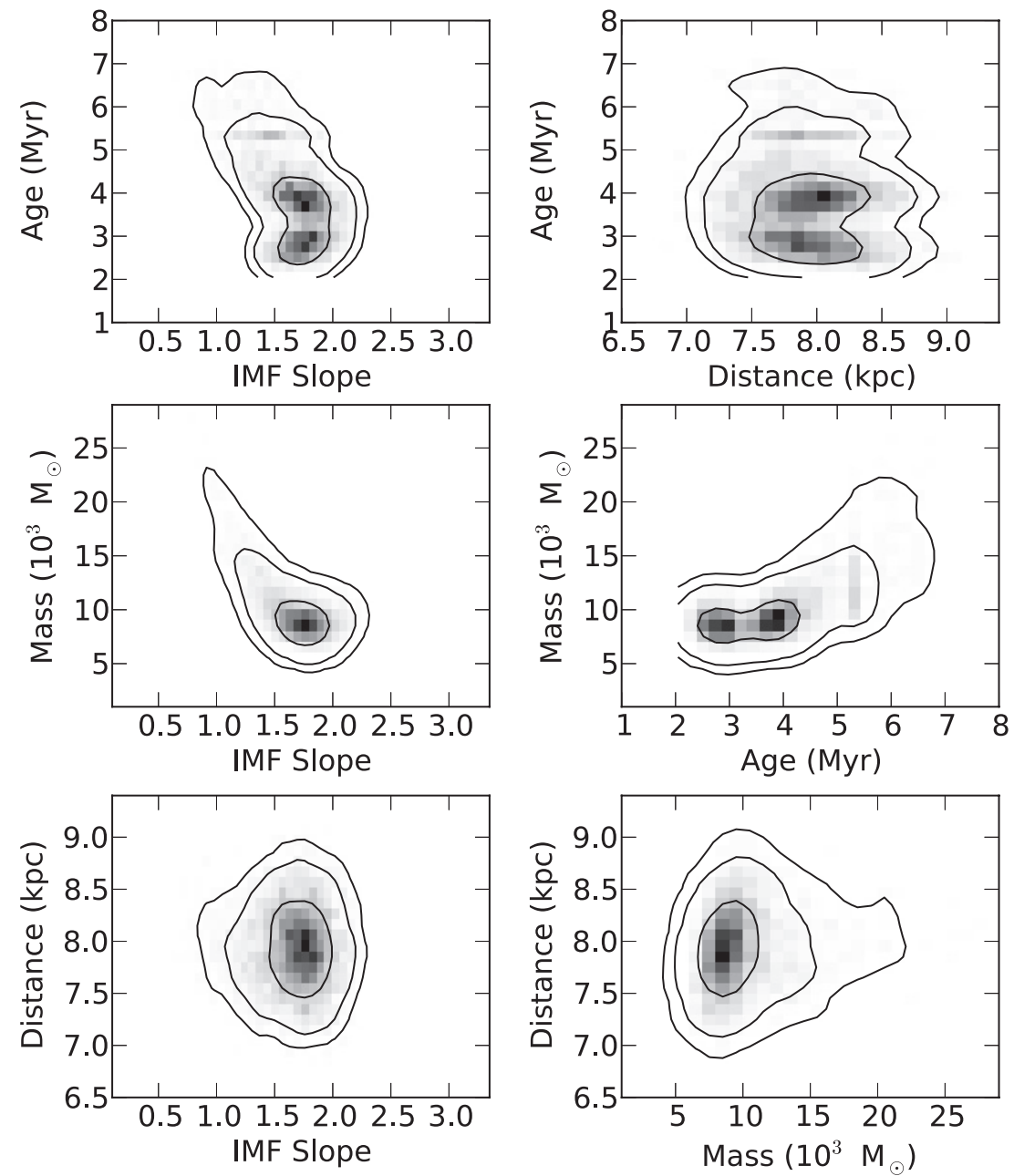

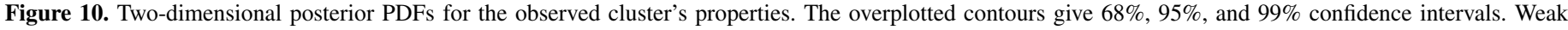

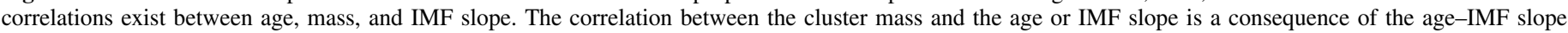

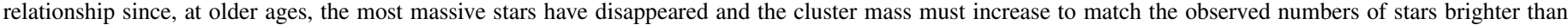
$K p=15.5$.

sample of young stars to a radius range of $0 .{ }^{\prime} 8-12^{\prime \prime}$ does not significantly change the KLF shape. Repeating our Bayesian analysis on this restricted sample produces a similar posterior probability distribution for the IMF slopes to that of our full sample.

We find a cluster that is well fit with an instantaneous star formation event at an age of 2.5-5.8 Myr with 95\% confidence. The most probable age is $3.9 \mathrm{Myr}$. This is smaller than the $6 \mathrm{Myr}$ commonly adopted, although consistent within the uncertainty range reported in Paumard et al. (2006). The age of the cluster is mainly constrained by the number of WR stars and the highest luminosity $\mathrm{OB}$ stars detected, since this effectively gives a main-sequence turnoff age. Careful examination of Figure 11 in Paumard et al. (2006) shows that best-fit ages, given the different numbers of $\mathrm{O}$ and WR stars (WN, WC, WNE, WNL), are actually either $4 \mathrm{Myr}$ or $8 \mathrm{Myr}$, with $6 \mathrm{Myr}$ being improbable for a Salpeter IMF. The inclusion of fainter OB stars in our spectroscopic sample substantially reduces the probability of cluster ages as old as $8 \mathrm{Myr}$. Figure 3 shows the expected number of WR and OB stars and their ratio as a function of cluster age for different assumed mass functions. While our Bayesian analysis captures uncertainties in the age due to random sampling of the mass function, it does not capture systematic uncertainties in models of stellar evolution. The lifetimes and luminosities of
WR stars are sensitive to metallicity (Fe and $\alpha$ elements), massloss rates, initial rotation rates, and close-binary mass exchange. Increased metallicity, mass-loss rates, rotation rates, and binary mass exchange all tend to increase the ages at which WR stars still exist (Eldridge \& Vink 2006; Meynet et al. 2006). On the other hand, increased metallicity reduces the main-sequence lifetimes. Furthermore, massive clusters, such as Westerlund 1, contain WR stars, blue supergiants, yellow hypergiants, and red supergiants at an age of $5 \mathrm{Myr}$; which no stellar evolution model successfully predicts (e.g., Negueruela et al. 2010). Therefore, we caution that ages up to 8 Myr may still be reasonable given current uncertainties in massive-star evolutionary models. We note that the IMF slope measurement is dominated by the relative luminosity distribution of $\mathrm{OB}$ main-sequence and supergiant stars, rather than the numbers of windy WR stars, and is far less sensitive to systematic uncertainties in evolutionary models. If the YNC does have a slightly younger age of $~ 4 \mathrm{Myr}$, then there are important implications for the dynamical history of the cluster. For instance, models of in situ star formation with a circular gas disk as the initial condition require at least $6 \mathrm{Myr}$ for the orbital eccentricities to evolve from circular to the observed eccentricity distribution peaked at $e=0.3$, assuming a Salpeter IMF (Alexander et al. 2007; Cuadra et al. 2008). Revised calculations are necessary to determine whether 

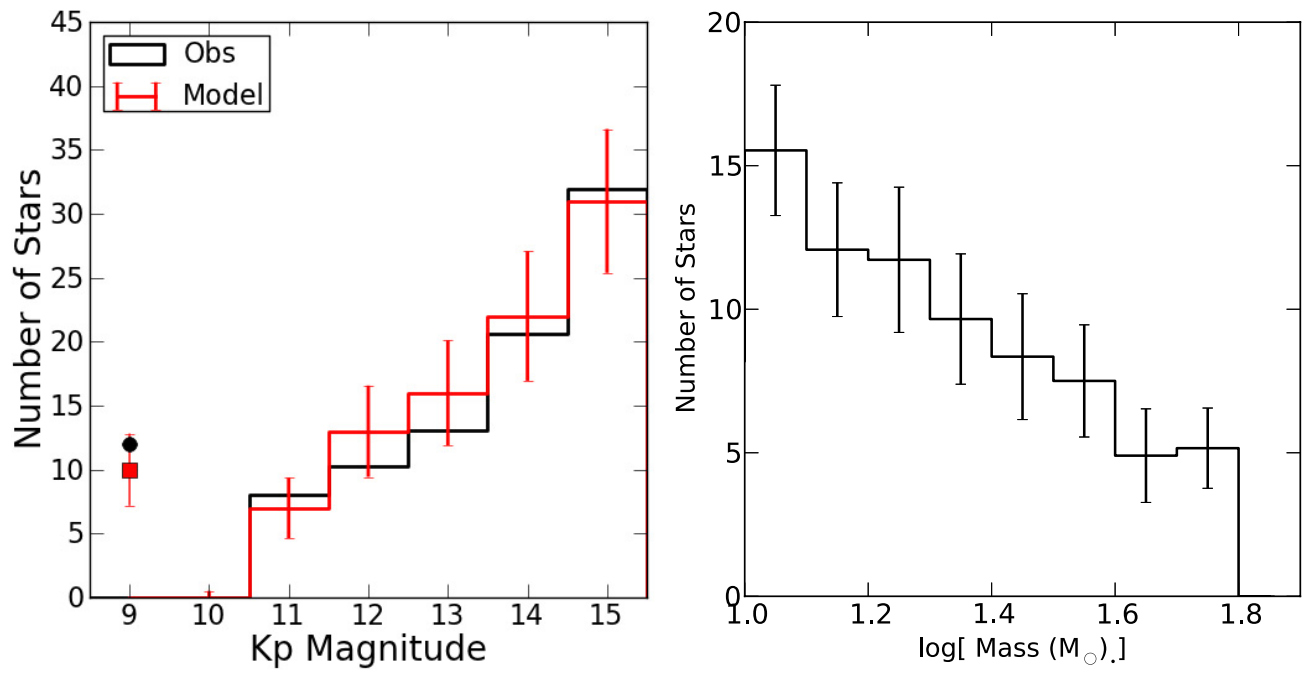

Figure 11. Observed (black) and model (red) $K p$-band luminosity function (left) and the resulting mass function (right). The model cluster includes multiple systems and has the properties listed in Table 2 (Solution 1). Left: the observed KLF (black line) and number of WR stars (black circle) show good agreement with the model KLF (red line) and model WR stars (red square). The observed $K p$ magnitudes (red) are corrected for differential extinction to a common extinction value of $A_{K s}=2.7$. The model KLF is taken as the mean and standard deviation (red error bars) of 100 simulated clusters, all with the same parameters, in order to account for variations due to stochastic sampling of the IMF. The imaging completeness curve is applied to each simulated cluster for direct comparison to the observed KLF. Right: the resulting mass function is constructed by converting the observed stars' $K p$ magnitudes to masses using the best-fit isochrone. To capture uncertainties in the stars' brightness, each star's $K p$ magnitude is randomly sampled 100 times from a Gaussian distribution centered on the measured $K p$ with a width of $\sigma_{K p}$. The resulting masses are binned into a mass function and the mean and standard deviation of the 100 mass functions are shown as a black line with error bars. The WR stars are not included in the mass function.

(A color version of this figure is available in the online journal.)
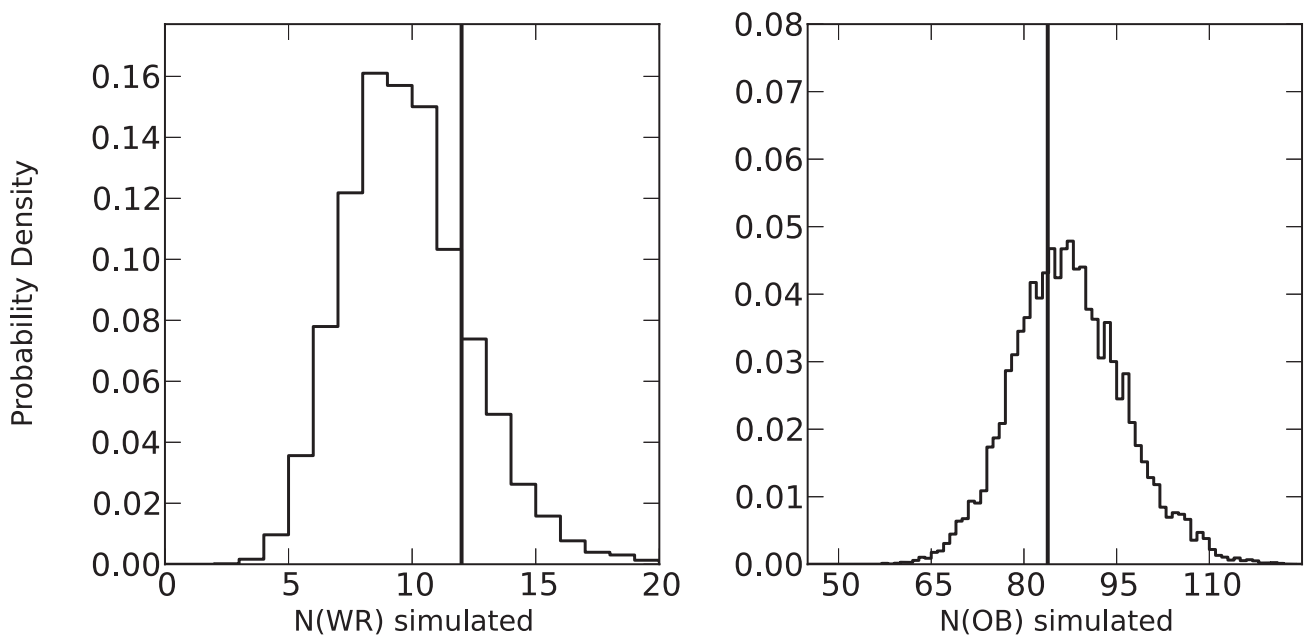

Figure 12. Comparison of the observed vs. inferred distributions for the number of WR stars (left) and the number of OB stars (right). The observed $N_{\mathrm{WR}}$ and $N_{\mathrm{OB}}$ are shown as vertical black lines and the probability distributions from the Bayesian analysis are shown as histograms.

the observed IMF slope and younger age can give rise to the observed high eccentricities in such a short time.

Ultimately, a complete spectroscopic and astrometric census of the young population in the YNC is required to accurately measure the recent star formation history and determine whether the distinct kinematic components were formed under different conditions, resulting in different mass functions. Expanding the survey area with current facilities will help clarify whether young stars on and off the disk have a different IMF. The Bayesian methodology presented here should be expanded to incorporate additional information from spectroscopy, such as effective temperature and gravity constraints for the OB stars, surface-abundance subtypes for the WR stars, and luminosities/ temperatures from WR wind/atmosphere modeling such as that presented in Martins et al. (2007). This additional information may improve age constraints, although uncertainties in stellar evolution for massive stars will still be a major factor. In the future, detection of pre-main-sequence turn-on points at $K p=17.5$ would more definitively constrain the age and star formation history. Furthermore, to truly constrain physical models of star formation at the Galactic center relative to local star formation processes, we will need measurements of the IMF shape over a broader mass range, ideally including any turnover suggesting a characteristic mass scale (e.g., $\sim 0.5 M_{\odot}$ in Orion). Some theories of star formation suggest that in extreme conditions, such as those found at the Galactic center, the characteristic mass may be significantly higher than in local starforming regions (Morris 1993; Bonnell et al. 2004; Krumholz $\&$ McKee 2008). Measurements of both the characteristic mass and the pre-main-sequence turn-on point should be possible with 

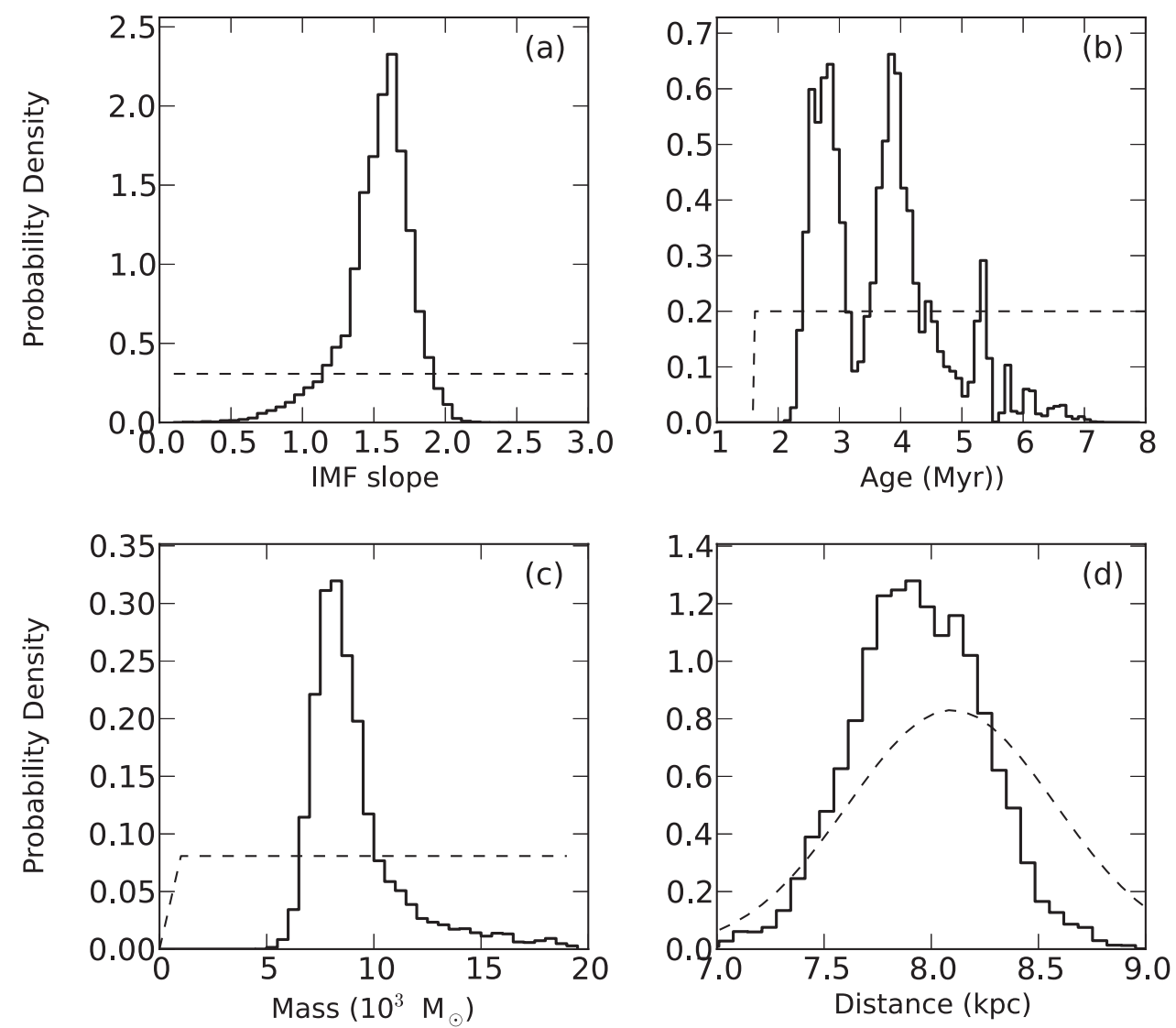

Figure 13. Same as Figure 9, but excluding stars with $r<0 . \prime 8$ to better match the sample chosen in Bartko et al. (2010). The peak of the probability distribution for the IMF slope shifts to $\alpha=1.6$; however, it is consistent with the result from the complete sample, within the $68 \%$ uncertainties. IMF slopes as top-heavy as $\alpha=0.45$ remain inconsistent at $99.89 \%$ probability. Ages of 6-7 Myr become slightly more probable.
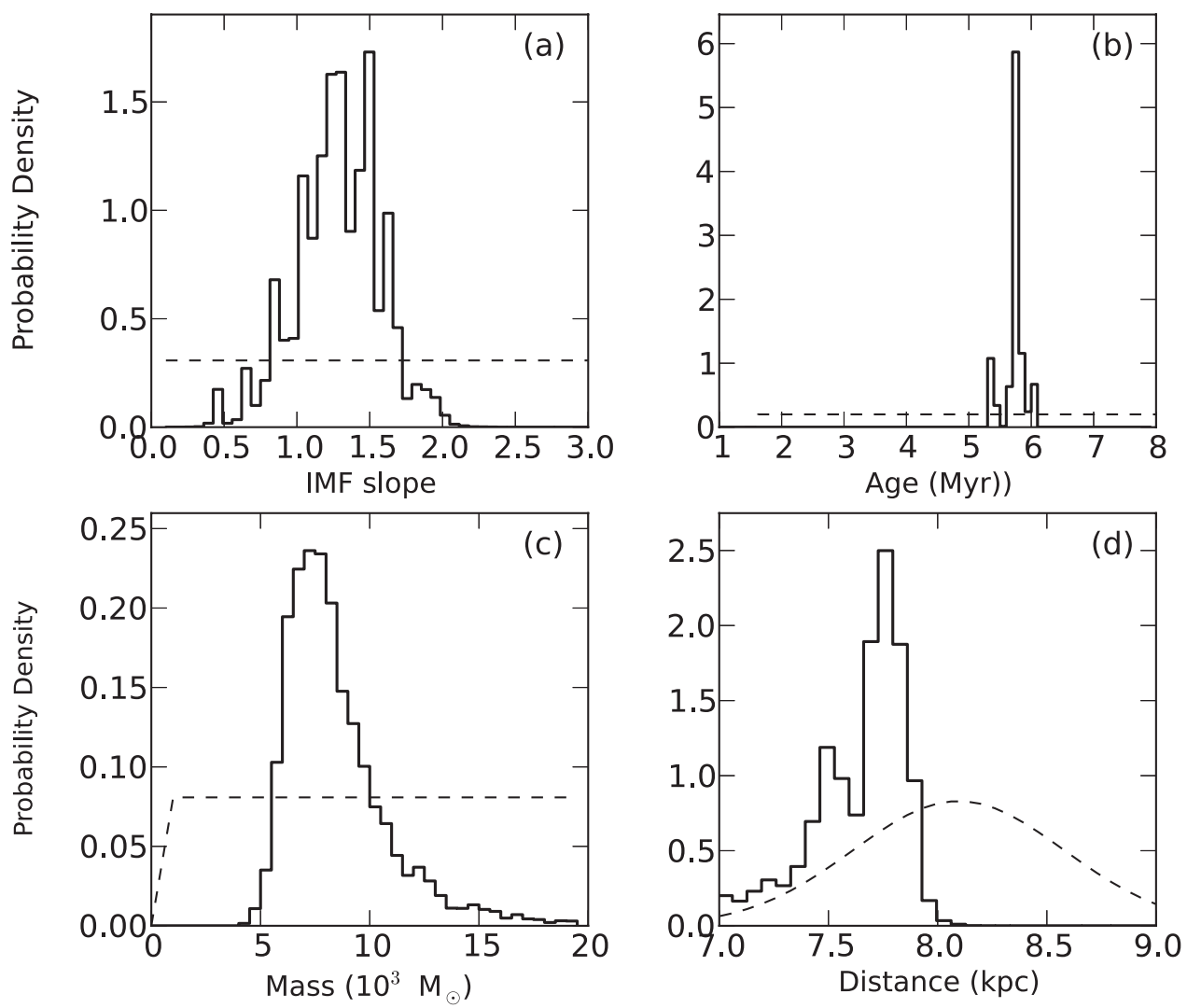

Figure 14. Same as Figure 9, but only allowing single stars in the model. The overall fit of the single-star model is poor and produces a strong bias away from the measured distance of $8 \mathrm{kpc}$. Furthermore, the resulting evidence for this model is substantially lower than for the model that includes multiples. 


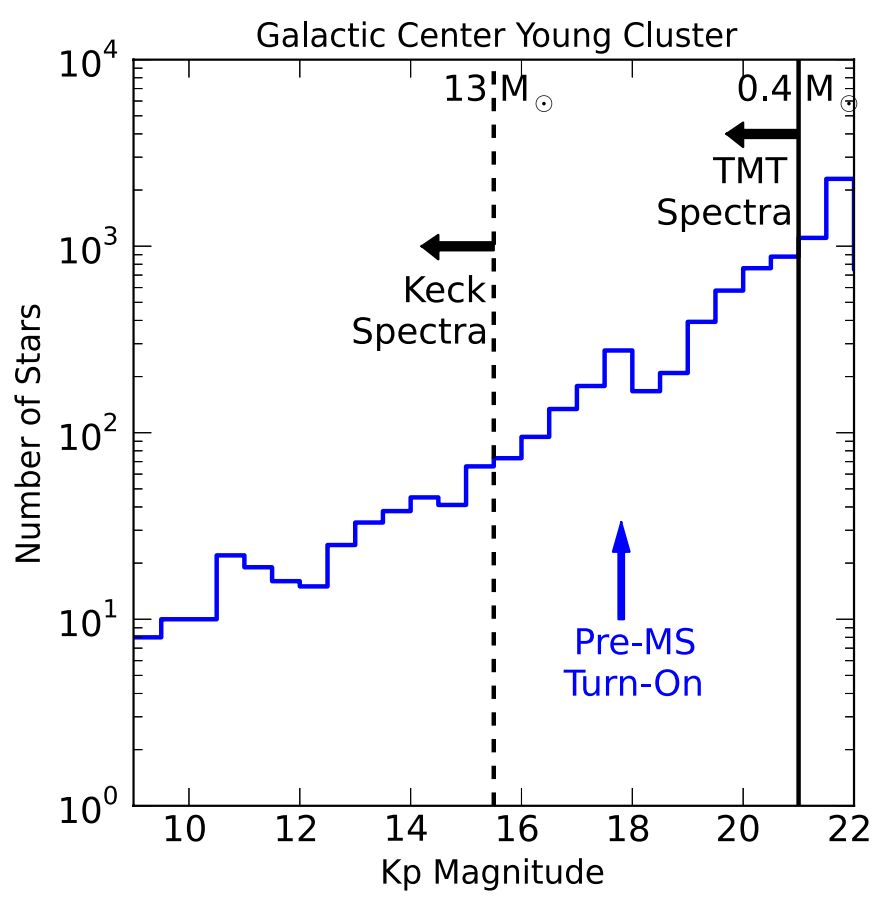

Figure 15. Model $K p$-band luminosity function for the YNC showing the relative sensitivity of Keck spectroscopy $(K p<15.5)$ and future TMT+IRIS spectroscopy $(K p<21)$. With TMT+IRIS, the pre-main-sequence turn-on point will be detectable at $K p \sim 17.5$ as well as the full IMF shape down to $\sim 0.4 M_{\odot}$. The model cluster shown here has an IMF slope of 1.7 down to $0.5 M_{\odot}$ and then turns over at $0.5 M_{\odot}$ to a slope of 1.3 down to $0.1 M_{\odot}$ as suggested by Weidner \& Kroupa (2004) for star formation in the local neighborhood. The model cluster has a distance of $8 \mathrm{kpc}$ and an extinction of $A_{K s}=2.7$.

(A color version of this figure is available in the online journal.)

the next generation of large ground-based telescopes equipped with adaptive optics-fed integral field spectrographs. One such instrument is IRIS on the Thirty Meter Telescope (TMT), which can accurately spectral-type stars in the Galactic center down to $K p=21$ in $1 \mathrm{hr}$ (Wright et al. 2010), as shown in Figure 15. Predicted IRIS sensitivities reach $0.4 M_{\odot}$ stars $(K p=21)$; however, distinguishing the young low-mass objects from the sea of much older main-sequence stars may require substantially higher signal-to-noise ratio to make precise measurements of effective temperatures. Such observations will be essential as the YNC is one of the few environments where significant deviations have been found from an otherwise near-universal IMF.

\section{CONCLUSIONS}

The Galactic center hosts a young nuclear star cluster around the SMBH. We use membership probabilities derived from spectroscopy, precise infrared photometry, and Bayesian inference methods to determine the global properties of the cluster. The best-fit age of the cluster is 3.9 Myr, somewhat younger than previously reported. The cluster's total mass extrapolated down to $1 M_{\odot}$ is between 14,000 and $37,000 M_{\odot}$ after correcting for incomplete azimuthal coverage. The best-fit IMF slope of 1.7 is flatter than a traditional Salpeter IMF, but far steeper than previously claimed in the literature. Future spectroscopic observations covering a larger field of view and extending to lower masses are necessary to understand the impact of mass segregation on IMF slope measurements and to determine whether the peak in the IMF also shows any significant difference from nearby star-forming regions.
We thank Greg Martinez for suggesting the MultiNest program and for helpful suggestions on our statistical analysis. J.R.L. acknowledges support from the California Institute of Technology Millikan Postdoctoral Fellow program and the NSF Astronomy and Astrophysics Postdoctoral Fellow program (AST-1102791). We also acknowledge support from NSF grant AST-0909218 (PI: A.M.G.) and the Levine-Leichtman family foundation. The data presented herein were obtained at the W. M. Keck Observatory, which is operated as a scientific partnership among the California Institute of Technology, the University of California and the National Aeronautics and Space Administration. The Observatory was made possible by the generous financial support of the W. M. Keck Foundation. The authors wish to recognize and acknowledge the very significant cultural role and reverence that the summit of Mauna Kea has always had within the indigenous Hawaiian community. We are most fortunate to have the opportunity to conduct observations from this mountain.

Facilities: Keck:II (NIRC2, OSIRIS)

\section{APPENDIX MULTIPLICITY}

The distribution of multiple star systems can be described completely by equations for the multiplicity frequency (MF), the companion star frequency (CSF), the mass ratio $(q)$, and the separation distribution. For star clusters at the distance of the Galactic center $(8 \mathrm{kpc})$, multiple systems are spatially unresolved and the separation distribution can be integrated over. The MF and CSF are defined as in Reipurth \& Zinnecker (1993)

$$
\begin{gathered}
\mathrm{MF}=\frac{B+T+Q+\cdots}{S+B+T+Q+\cdots} \\
\mathrm{CSF}=\frac{B+2 T+3 Q+\cdots}{S+B+T+Q+\cdots}
\end{gathered}
$$

where $S$ is the number of single stars, $B$ is the number of binaries, $T$ is the number of triples, and $Q$ is the number of quadruples. The multiplicity fraction always ranges between 0 and 1 , but the companion star fraction, which is the mean number of companions, can be greater than 1, such as for the Orion Trapezium stars where CSF > 1.5 (Preibisch et al. 1999; Zinnecker \& Yorke 2007). Based on observations, the MF and CSF are known to vary with primary star mass, the age of a cluster, and possibly the density of a cluster (e.g., Ghez et al. 1993; Petr et al. 1998; Mason et al. 1998, 2009; Duchêne et al. 1999, 2007; Köhler et al. 2000, 2006, 2008; Reipurth 2000; Patience et al. 2002; Shatsky \& Tokovinin 2002; Raghavan et al. 2010; Kraus \& Hillenbrand 2012). Complete multiplicity surveys of young clusters $(<10 \mathrm{Myr})$ that span a large range of primary stellar masses $\left(0.1->10 M_{\odot}\right)$, mass ratios, and separations are difficult to conduct and only a few exist in the literature (Lafrenière et al. 2008; Kobulnicky \& Fryer 2007; Kouwenhoven et al. 2007). Star formation theories do not yet predict an analytic functional form for the MF, CSF, and $q$ distributions and how they vary with primary mass; however, simulations are beginning to produce distributions that are in rough agreement with observations (Bate 2012; Krumholz et al. 2012). Therefore, we take an empirical approach and compile measurements of the MF and CSF as a function of mass from published surveys of young clusters (<10 Myr), which span 


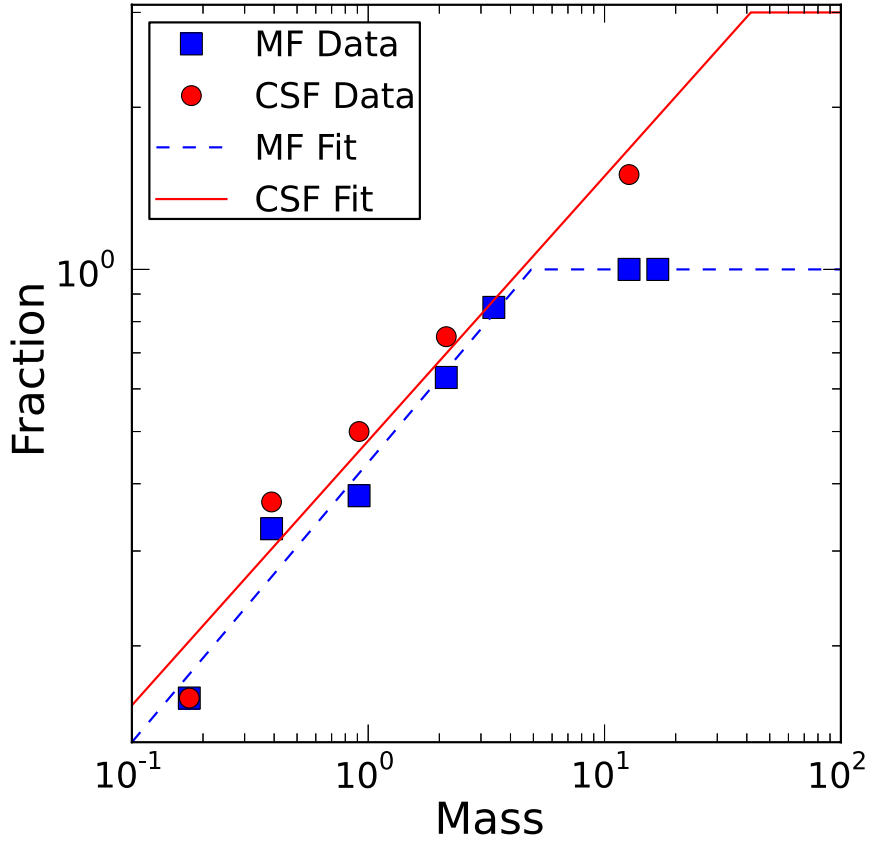

Figure 16. Multiplicity frequency (MF) and the companion star frequency (CSF) as a function of primary mass. Empirical measurements are plotted for the MF (blue squares) and CSF (red circles) values reported in the literature (Table 3). The data are fit with power laws (lines) as given by Equations (A3) and (A4). The CSF is truncated at $\leqslant 3$, which impacts systems with a primary mass above $\sim 40 M_{\odot}$.

(A color version of this figure is available in the online journal.)

Table 3

Multiplicity Measurements for Young Clusters

\begin{tabular}{lccl}
\hline \hline $\begin{array}{l}\text { Mass } \\
\left(M_{\odot}\right)\end{array}$ & MF & CSF & \multicolumn{1}{c}{ Reference } \\
\hline 0.175 & 0.16 & 0.16 & Cha I; Lafrenière et al. (2008) \\
0.390 & 0.33 & 0.37 & Cha I; Lafrenière et al. (2008) \\
0.915 & 0.38 & 0.50 & Cha I; Lafrenière et al. (2008) \\
2.14 & 0.63 & 0.75 & Cha I; Lafrenière et al. (2008) \\
3.4 & 0.85 & $\ldots$ & Sco OB2; Kouwenhoven et al. (2007) \\
12.7 & 1.00 & 1.5 & Orion (ONC); Preibisch et al. (1999) \\
16.8 & 1.00 & $\ldots$ & Cyg OB2; Kobulnicky \& Fryer (2007) \\
\hline
\end{tabular}

Notes.

a Mean mass of the sample was estimated from Rizzuto et al. (2011).

b Mean mass of the sample was estimated from Kiminki et al. (2007).

stellar masses of $0.2-17 M_{\odot}$ and all separations (Table 3). This compilation is incomplete as we exclude surveys that only report multiplicity for masses below $1 M_{\odot}$ and those without CSF information. The above high-mass limit is set by the available multiplicity surveys. and the low-mass cutoff is chosen as a reasonable detection threshold for stars in more distant massive young clusters with both current (e.g., Keck) and future (e.g., TMT) spectroscopy. The compiled MF and CSF data (Figure 16) are fit with a power-law dependence on mass and give the following results:

$$
\begin{array}{ccc}
\mathrm{MF}(m)=A m^{\gamma} & A=0.44 & \gamma=0.51 \\
\mathrm{CSF}(m)=B m^{\beta} & B=0.50 & \beta=0.45 .
\end{array}
$$

The best-fit power laws are also shown in Figure 16. For any star cluster older than a few crossing times, the CSF will not continue to grow indefinitely at high masses due to the dynamical instability of high-order systems in a clustered environment. A hard limit to the mean number of companions is adopted (CSF $\leqslant 3$ ) such that systems with more than three components are allowed, but have a low probability of occurrence. Lastly, some observations suggest that multiplicity properties may evolve over time differently for clusters with different masses or stellar densities (e.g., Kroupa 1995; Duchêne et al. 1999; Köhler et al. 2006; Marks \& Kroupa 2012). However, the completeness and significance of these results is still uncertain (King et al. 2012) and we therefore assume all young clusters $(<10 \mathrm{Myr})$ have the same multiplicity properties, including the YNC.

\section{REFERENCES}

Alexander, R. D., Armitage, P. J., Cuadra, J., \& Begelman, M. C. 2008, ApJ, 674, 927

Alexander, R. D., Begelman, M. C., \& Armitage, P. J. 2007, ApJ, 654, 907

Allard, F., Hauschildt, P. H., \& Schwenke, D. 2000, ApJ, 540, 1005

Allen, D. A., Hyland, A. R., \& Hillier, D. J. 1990, MNRAS, 244, 706

Allen, P. R., Koerner, D. W., Reid, I. N., \& Trilling, D. E. 2005, ApJ, 625, 385

Bartko, H., Martins, F., Fritz, T. K., et al. 2009, ApJ, 697, 1741

Bartko, H., Martins, F., Trippe, S., et al. 2010, ApJ, 708, 834

Baruteau, C., Cuadra, J., \& Lin, D. N. C. 2011, ApJ, 726, 28

Bate, M. R. 2012, MNRAS, 419, 3115

Bender, R., Kormendy, J., Bower, G., et al. 2005, ApJ, 631, 280

Blum, R. D., Depoy, D. L., \& Sellgren, K. 1995, ApJ, 441, 603

Bonnell, I. A., Vine, S. G., \& Bate, M. R. 2004, MNRAS, 349, 735

Bridges, M., Feroz, F., Hobson, M. P., \& Lasenby, A. N. 2009, MNRAS, 400, 1075

Brown, W. R., Geller, M. J., Kenyon, S. J., Kurtz, M. J., \& Bromley, B. C. 2007, ApJ, 671, 1708

Carr, J. S., Sellgren, K., \& Balachandran, S. C. 2000, ApJ, 530, 307

Castelli, F., \& Kurucz, R. L. 2003, in IAU Symp. 210, Modelling of Stellar Atmospheres, ed. N. Piskunov, W. W. Weiss, \& D. F. Gray (Cambridge: Cambridge Univ. Press), A20

Converse, J. M., \& Stahler, S. W. 2008, ApJ, 678, 431

Cuadra, J., Armitage, P. J., \& Alexander, R. D. 2008, MNRAS, 388, L64

Cunha, K., Sellgren, K., Smith, V. V., et al. 2007, ApJ, 669, 1011

De Gennaro, S., von Hippel, T., Jefferys, W. H., et al. 2009, ApJ, 696, 12

Do, T., Lu, J. R., Ghez, A. M., et al. 2013, ApJ, 764, 154

Duchêne, G., Bontemps, S., Bouvier, J., et al. 2007, A\&A, 476, 229

Duchêne, G., Bouvier, J., \& Simon, T. 1999, A\&A, 343, 831

Eckart, A., \& Genzel, R. 1997, MNRAS, 284, 576

Eisenhauer, F., Genzel, R., Alexander, T., et al. 2005, ApJ, 628, 246

Ekström, S., et al. 2012, A\&A, 537, A146

Eldridge, J. J., \& Vink, J. S. 2006, A\&A, 452, 295

Espinoza, P., Selman, F. J., \& Melnick, J. 2009, A\&A, 501, 563

Feroz, F., \& Hobson, M. P. 2008, MNRAS, 384, 449

Feroz, F., Hobson, M. P., \& Bridges, M. 2009, MNRAS, 398, 1601

Genzel, R., Pichon, C., Eckart, A., Gerhard, O. E., \& Ott, T. 2000, MNRAS, 317,348

Genzel, R., Schödel, R., Ott, T., et al. 2003, ApJ, 594, 812

Gerhard, O. 2001, ApJL, 546, L39

Ghez, A. M., Duchêne, G., Matthews, K., et al. 2003, ApJL, 586, L127

Ghez, A. M., Klein, B. L., Morris, M., \& Becklin, E. E. 1998, ApJ, 509, 678

Ghez, A. M., Morris, M., Becklin, E. E., Tanner, A., \& Kremenek, T. 2000, Natur, 407,349

Ghez, A. M., Neugebauer, G., \& Matthews, K. 1993, AJ, 106, 2005

Ghez, A. M., Salim, S., Hornstein, S. D., et al. 2005, ApJ, 620, 744

Ghez, A. M., Salim, S., Weinberg, N. N., et al. 2008, ApJ, 689, 1044

Gillessen, S., Eisenhauer, F., Fritz, T. K., et al. 2009a, ApJL, 707, L114

Gillessen, S., Eisenhauer, F., Trippe, S., et al. 2009b, ApJ, 692, 1075

Goodman, J. 2003, MNRAS, 339, 937

Goodwin, S. P., \& Kroupa, P. 2005, A\&A, 439, 565

Gürkan, M. A., \& Rasio, F. A. 2005, ApJ, 628, 236

Haas, J., Šubr, L., \& Kroupa, P. 2011, MNRAS, 412, 1905

Haas, M. R. 2010, PhD thesis, Univ. Leiden

Hamann, W.-R., Gräfener, G., Feldmeier, A., et al. 2008, in ASP Conf. Ser. 391, Hydrogen-Deficient Stars, ed. A. Werner \& T. Rauch (San Francisco, CA: ASP), 293

Hansen, B. M. S., \& Milosavljević, M. 2003, ApJL, 593, L77

Hauschildt, P. H., Allard, F., \& Baron, E. 1999, ApJ, 512, 377

Hobbs, A., \& Nayakshin, S. 2009, MNRAS, 394, 191

Hobson, M. P., Jaffe, A. H., Liddle, A. R., Mukeherjee, P., \& Parkinson, D. (ed.) 2010, Bayesian Methods in Cosmology (New York: Cambridge Univ. Press) 
Kilbinger, M., Wraith, D., Robert, C. P., et al. 2010, MNRAS, 405, 2381

Kim, S. S., Figer, D. F., \& Morris, M. 2004, ApJL, 607, L123

Kim, S. S., \& Morris, M. 2003, ApJ, 597, 312

Kiminki, D. C., \& Kobulnicky, H. A. 2012, ApJ, 751, 4

Kiminki, D. C., Kobulnicky, H. A., Kinemuchi, K., et al. 2007, ApJ, 664, 1102

King, R. R., Parker, R. J., Patience, J., \& Goodwin, S. P. 2012, MNRAS, 421, 2025

Kobulnicky, H. A., \& Fryer, C. L. 2007, ApJ, 670, 747

Köhler, R., Kunkel, M., Leinert, C., \& Zinnecker, H. 2000, A\&A, 356, 541

Köhler, R., Neuhäuser, R., Krämer, S., et al. 2008, A\&A, 488, 997

Köhler, R., Petr-Gotzens, M. G., McCaughrean, M. J., et al. 2006, A\&A, 458,461

Kolykhalov, P. I., \& Syunyaev, R. A. 1980, SvAL, 6, 357

Kouwenhoven, M. B. N., Brown, A. G. A., Goodwin, S. P., Portegies Zwart, S. F., \& Kaper, L. 2009, A\&A, 493, 979

Kouwenhoven, M. B. N., Brown, A. G. A., Portegies Zwart, S. F., \& Kaper, L. 2007, A\&A, 474, 77

Krabbe, A., Genzel, R., Drapatz, S., \& Rotaciuc, V. 1991, ApJL, 382, L19

Krabbe, A., Genzel, R., Eckart, A., et al. 1995, ApJL, 447, L95

Kraus, A. L., \& Hillenbrand, L. A. 2012, ApJ, 757, 141

Kroupa, P. 1995, MNRAS, 277, 1491

Krumholz, M. R., Klein, R. I., \& McKee, C. F. 2012, ApJ, 754, 71

Krumholz, M. R., \& McKee, C. F. 2008, Natur, 451, 1082

Lafrenière, D., Jayawardhana, R., Brandeker, A., Ahmic, M., \& van Kerkwijk, M. H. 2008, ApJ, 683, 844

Lauer, T. R., Faber, S. M., Ajhar, E. A., Grillmair, C. J., \& Scowen, P. A. 1998, AJ, 116, 2263

Levin, Y., \& Beloborodov, A. M. 2003, ApJL, 590, L33

Löckmann, U., Baumgardt, H., \& Kroupa, P. 2009, MNRAS, 398, 429

Lu, J. R., Ghez, A. M., Hornstein, S. D., Morris, M., \& Becklin, E. E. 2005, ApJL, 625, L51

Lu, J. R., Ghez, A. M., Hornstein, S. D., et al. 2009, ApJ, 690, 1463

Madigan, A.-M., Hopman, C., \& Levin, Y. 2011, ApJ, 738, 99

Maillard, J. P., Paumard, T., Stolovy, S. R., \& Rigaut, F. 2004, A\&A, 423, 155

Maíz Apellániz, J. 2008, ApJ, 677, 1278

Maíz-Apellániz, J. 2009, Ap\&SS, 324, 95

Maíz Apellániz, J., \& Úbeda, L. 2005, ApJ, 629, 873

Marks, M., \& Kroupa, P. 2012, A\&A, 543, A8

Martinez, G. D., Minor, Q. E., Bullock, J., et al. 2011, ApJ, 738, 55

Martins, F., Genzel, R., Hillier, D. J., et al. 2007, A\&A, 468, 233

Martins, F., Gillessen, S., Eisenhauer, F., et al. 2008, ApJL, 672, L119

Mason, B. D., Hartkopf, W. I., Gies, D. R., Henry, T. J., \& Helsel, J. W. 2009, AJ, 137,3358

Mason, B. D., Henry, T. J., Hartkopf, W. I., ten Brummelaar, T., \& Soderblom, D. R. 1998, AJ, 116, 2975

McMillan, S. L. W., \& Portegies Zwart, S. F. 2003, ApJ, 596, 314

Meynet, G., \& Maeder, A. 2003, A\&A, 404, 975

Meynet, G., Mowlavi, N., \& Maeder, A. 2006, arXiv:astro-ph/0611261

Morris, M. 1993, ApJ, 408, 496

Morris, M., \& Serabyn, E. 1996, ARA\&A, 34, 645

Najarro, F., Figer, D. F., Hillier, D. J., \& Kudritzki, R. P. 2004, ApJL, 611, L105

Najarro, F., Krabbe, A., Genzel, R., et al. 1997, A\&A, 325, 700
Nayakshin, S. 2006, MNRAS, 372, 143

Nayakshin, S., \& Cuadra, J. 2005, A\&A, 437, 437

Nayakshin, S., Cuadra, J., \& Springel, V. 2007, MNRAS, 379, 21

Nayakshin, S., Dehnen, W., Cuadra, J., \& Genzel, R. 2006, MNRAS, 366,1410

Nayakshin, S., \& Sunyaev, R. 2005, MNRAS, 364, L23

Negueruela, I., Clark, J. S., \& Ritchie, B. W. 2010, A\&A, 516, A78

Nishiyama, S., Tamura, M., Hatano, H., et al. 2009, ApJ, 696, 1407

Patience, J., Ghez, A. M., Reid, I. N., \& Matthews, K. 2002, AJ, 123, 1570

Paumard, T., Genzel, R., Martins, F., et al. 2006, ApJ, 643, 1011

Perets, H. B., Hopman, C., \& Alexander, T. 2007, ApJ, 656, 709

Petr, M. G., Coudé du Foresto, V., Beckwith, S. V. W., Richichi, A., \& McCaughrean, M. J. 1998, ApJ, 500, 825

Portegies Zwart, S. F., McMillan, S. L. W., \& Gerhard, O. 2003, ApJ, 593, 352

Preibisch, T., Balega, Y., Hofmann, K.-H., Weigelt, G., \& Zinnecker, H. 1999, New A, 4, 531

Press, W. H. 1997, in Unsolved Problems in Astrophysics, ed. J. N. Bahcall \& J. P. Ostriker (Princeton, NJ: Princeton Univ. Press), 49

Raghavan, D., McAlister, H. A., Henry, T. J., et al. 2010, ApJS, 190, 1

Ramírez, S. V., Sellgren, K., Carr, J. S., et al. 2000, ApJ, 537, 205

Reipurth, B. 2000, AJ, 120, 3177

Reipurth, B., \& Zinnecker, H. 1993, A\&A, 278, 81

Rizzuto, A. C., Ireland, M. J., \& Robertson, J. G. 2011, MNRAS, 416, 3108

Sagar, R., \& Richtler, T. 1991, A\&A, 250, 324

Salpeter, E. E. 1955, ApJ, 121, 161

Sanders, R. H. 1998, MNRAS, 294, 35

Schaller, G., Schaerer, D., Meynet, G., \& Maeder, A. 1992, A\&AS, 96, 269

Schödel, R., Eckart, A., Iserlohe, C., Genzel, R., \& Ott, T. 2005, ApJL, 625, L111

Schödel, R., Najarro, F., Muzic, K., \& Eckart, A. 2010, A\&A, 511, A18

Schödel, R., Ott, T., Genzel, R., et al. 2002, Natur, 419, 694

Schödel, R., Ott, T., Genzel, R., et al. 2003, ApJ, 596, 1015

Selman, F., Melnick, J., Bosch, G., \& Terlevich, R. 1999, A\&A, 347, 532

Seth, A. C., Dalcanton, J. J., Hodge, P. W., \& Debattista, V. P. 2006, AJ, 132,2539

Shatsky, N., \& Tokovinin, A. 2002, A\&A, 382, 92

Shlosman, I., \& Begelman, M. C. 1989, ApJ, 341, 685

Siess, L., Dufour, E., \& Forestini, M. 2000, A\&A, 358, 593

Skilling, J. 2004, in AIP Conf. Proc. 735, Bayesian Inference and Maximum Entropy Methods in Science and Engineering, ed. R. Fischer, R. Preuss, \& U. V. Toussaint (Melville, NY: AIP), 395

Tamblyn, P., Rieke, G. H., Hanson, M. M., et al. 1996, ApJ, 456, 206

Thies, I., \& Kroupa, P. 2007, ApJ, 671, 767

van Dyk, D. A., Degennaro, S., Stein, N., Jefferys, W. H., \& von Hippel, T. 2009, AnApS, 3, 117

Veitch, J., \& Vecchio, A. 2010, PhRvD, 81, 062003

Vollmer, B., \& Duschl, W. J. 2001, A\&A, 377, 1016

Weidner, C., \& Kroupa, P. 2004, MNRAS, 348, 187

Weidner, C., Kroupa, P., \& Maschberger, T. 2009, MNRAS, 393, 663

Wright, S. A., Barton, E. J., Larkin, J. E., et al. 2010, Proc. SPIE, 7735, 77357P

Yelda, S. 2012, PhD thesis, Univ. California, Los Angeles

Zinnecker, H., \& Yorke, H. W. 2007, ARA\&A, 45, 481 